\title{
The application of joint sparsity and total variation minimization algorithms to a real-life art restoration problem
}

\author{
Massimo Fornasier* \\ Ronny Ramlau ${ }^{\dagger}$ \\ Gerd Teschke
}

October 7, 2008

\begin{abstract}
On March 11, 1944, the famous Eremitani Church in Padua (Italy) was destroyed in an Allied bombing along with the inestimable frescoes by Andrea Mantegna et al. contained in the Ovetari Chapel. In the last 60 years, several attempts have been made to restore the fresco fragments by traditional methods, but without much success. One of the authors contributed to the development of an efficient pattern recognition algorithm to map the original position and orientation of the fragments, based on comparisons with an old gray level image of the fresco prior to the damage. This innovative technique allowed for the partial reconstruction of the frescoes. Unfortunately, the surface covered by the colored fragments is only $77 \mathrm{~m}^{2}$, while the original area was of several hundreds. This means that we can reconstruct only a fraction (less than $8 \%$ ) of this inestimable artwork. In particular the original color of the blanks is not known. This begs the question of whether it is possible to estimate mathematically the original colors of the frescoes by making use of the potential information given by the available fragments and the gray level of the pictures taken before the damage. Moreover, is it possible to estimate how faithful such a restoration is? In this paper we retrace the development of the recovery of the frescoes as an inspiring and challenging real-life problem for the development of new mathematical methods. Then we shortly review two models recently studied independently by the authors for the recovery of vector valued functions from incomplete data, with applications to the recolorization problem. The models are based on the minimization of a functional which is formed by the discrepancy with respect to the data and additional regularization constraints. The latter refer to joint sparsity measures with respect to frame expansions, in particular wavelet or curvelet expansions, for the first functional and functional total variation for the second. We establish relations between these two models. As a major contribution of this work we perform specific numerical test on the real-life problem of the A. Mantegna's frescoes and we compare the results due to the two methods.
\end{abstract}

MSC: 15A29, 47A52, 68U10, 94A08, 94A40

Keywords: Recolorization, inpainting, wavelet regularization, TV regularization, multi-channel data, joint sparsity

\footnotetext{
* Johann Radon Institute for Computational and Applied Mathematics (RICAM), Austrian Academy of Sciences, Altenbergerstr. 69, 4040 Linz, Austria.

${ }^{\dagger}$ Johann Radon Institute for Computational and Applied Mathematics (RICAM), Austrian Academy of Sciences, Altenbergerstr. 69, 4040 Linz, Austria.

${ }^{\ddagger}$ Institute for Computational Mathematics in Science and Technology, University of Applied Sciences Neubrandenburg, Brodaer Str. 2, 17033 Neubrandenburg and as Junior Konrad-Zuse-Fellow with the Zuse-Institute Berlin, Germany.
} 


\section{Introduction}

\subsection{Mathematical imaging in art restoration}

We address the problem of the faithful reconstruction of vector valued functions from incomplete data, with special emphasis in color image recovery. We are inspired by a real-life problem, i.e. the rebirth of one of the most important masterpieces of the Italian Renaissance, by making use of mathematical imaging techniques. We refer to the decorative cycle in the Ovetari Chapel in the Eremitani Church in Padua. The chapel was seriously damaged by an air strike in 1944 and a large section of the contained frescoes were sparsely fragmented. A digital cataloging of pictures of the remaining fragments made it possible to count the number (78.561) of those with an area larger than $1 \mathrm{~cm}^{2}$. The distribution of the areas shows that most of them are relatively small $\left(5-6 \mathrm{~cm}^{2}\right)$. There is no information on the possible location of the pieces on the huge original surface and also unknown is the angle of rotation with respect to the original orientation. These a priori data demonstrated the lack of contiguous fragments for any given fragment. These difficulties explain the unsuccessful attempts of recomposition by traditional methods. In simple words, it is an incomplete puzzle which is too big to be solved by human eyes only. There exist some fairly good quality black and white photographs of the frescoes dated from between 1900 and 1920.This heritage gave rise to the hope that a computer-based comparison between the fresco digital images and those of the fragments could help to recognize their original location. The request of a fast algorithm excludes the implementation of comparisons pixel-by-pixel and suggests that methods based on compressed/sparse representations, i.e., basis or frame expansions, can be more efficient. In [33, 32] a specific pattern recognition algorithm based on sparse circular harmonic expansions was proposed. This method was implemented for the solution of the fragment recollocation problem and we illustrate some of the final results in Figure 1. On the basis of the map produced by this computer assisted anastylosis, parts of the frescoes have already been physically restored. We refer to the book chapter [9] for more details. Even though the collocation of one single fragment is of historical and cultural importance, the success of the computer assisted anastylosis was partially spoiled by the limited surface that the fragments can cover. This begs the question of whether it is possible to estimate mathematically the original colors of the missing parts of the frescoes by making use of the potential information given by the available fragments and the gray level of the pictures taken before the damage. Can one estimate how faithful such restoration is?

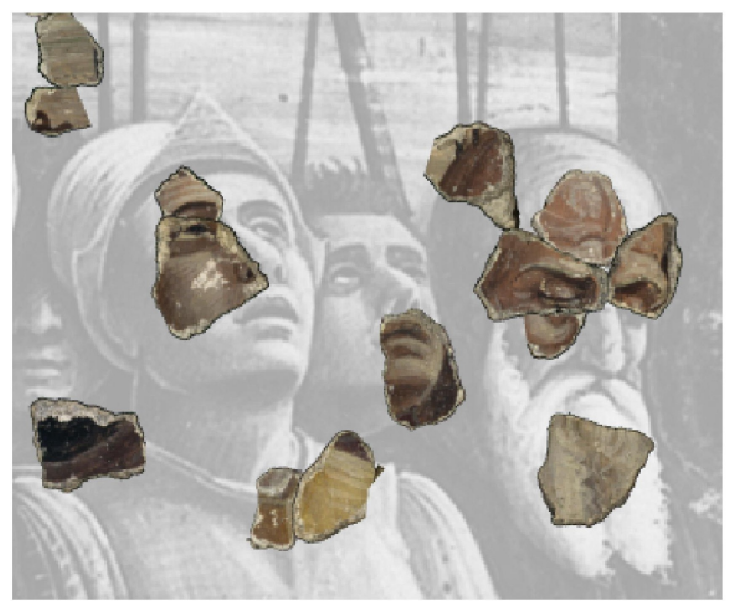

Figure 1: Fragmented A. Mantegna's frescoes (1452) by a bombing in the Second World War. Computer based reconstruction by using efficient pattern matching techniques [33]. 


\subsection{Mathematical inpainting and recolorization}

Mathematical inpainting, an artistic synonym for image interpolation, was introduced by Sapiro et al. [3] with the specific purpose of imitating the basic approaches used by professional restorers when filling blanks in paintings. Their algorithm amounts to the solution of an evolutionary differential equation whose steady-state is the prolongation of the incomplete image in the inpainting region to make constant the information along isophotes, for further recent developments see also [2]. Closely related to inpainting is the contribution by Masnou and Morel $[38,39]$ who addressed the so-called disocclusion problem. Essentially it amounts to an application of the principle of good continuation, i.e., without forming undesired T-junctions and abrupt direction changes, of the image level curves into the region where an occlusion occurred in order to restore the essential morphology. This work can be seen as a development of the theory of Euler's elastica curves by Mumford [40]. Chan and Shen contributed to inpainting with other models similar or related to the ones previously cited, see $[11,12,13,14]$ and also $[25,27,37]$. In simple words, mathematical inpainting is the attempt to guess the morphology of the image in a relatively small missing part from the level curves of the relevant known part. The recolorization problem, like that of the frescoes, can be viewed as a particular case of inpainting. Nevertheless, in this case two significant differences occur with respect to the classical problem: 1) The region of missing color is usually much larger than the one with known colors 2 ) the morphology of the image in the missing part can be determined by the known gray level, see also [7]. Several approaches to the recovery of colors in gray level images have been recently proposed based on different intuitions. Assuming that neighboring pixels with similar intensities should have similar color, an algorithm involving a non-local fitting term has been proposed in [35] to match the colors. Similarly, a fast algorithm using a weighted distance image blending technique is studied in [49]. From the assumption that the color morphology is essentially determined by the gradient of the gray level, Sapiro proposed in [44] a recolorization method based on minimizing the difference between the gradient of gray level and the gradient of color. The problem reduces to the solution of a (nonlinear) boundary value problem. Based on similar assumptions two variational approaches are proposed in [34] where the authors minimize the discrepancy with respect to the color datum and impose a smoothness constraint on the solution out of the gray level discontinuity set. All the proposed solutions show that a very limited amount of color is sufficient information to recover a pleasant result in ad hoc cases.

\subsection{Scope of the paper}

In this paper we shortly review two different approaches to recolorization previously proposed by the authors in $[28,29,45]$. Both the methods were tested on images where we knew the original color. In these cases, also our methods produced rather successful results which could be confirmed by comparison with the original images. Differently from previous contributions, in this paper we present the results of applications of these recolorization models to a real-life problem, the one of the A. Mantegna's frescoes. In this case, we face significant difficulties due to

(i) the unknown color-gray conversion,

(ii) the presence of a very strong noise with unknown statistical distribution,

(iii) and the fact that we have no way to compare the obtained results with an original color version of the frescoes.

Hence, a positive reconstruction result is indeed far beyond from being obvious. Beside the cultural and historical importance of the specific application, we want to emphasize that reallife experiments are usually rarely shown and the difficulties related to these experiments are 
usually underestimated in the mathematics community. Therefore we think it is an important task to step beyond academic examples and consider now real-life applications. In particular, point (iii) of the previous list of significant difficulties motivates us to the comparison of our proposed methods as a mutual benchmark of the results. Since the methods differ in certain aspects we hope that they can offer a complementary test of faithfulness.

\subsection{The general setting}

Since color images are modeled as multichannel signals, the problem is reformulated as the recovery of vector valued functions from incomplete data. The incomplete information is assumed as the result of a distortion, with values in a lower dimensional manifold. The vector components are assumed to be coupled. The difference between the proposed methods is the way we couple the information. For both the approaches, the recovery is realized as the minimization of a functional which is formed by the discrepancy with respect to the data and additional regularization constraints. The latter refer to joint sparsity measures with respect to frame expansions for the first functional and functional total variation for the second.

For a clearer understanding of the general setting, we now introduce some notations. Let $\Omega$ be an open, bounded, and connected subset of $\mathbb{R}^{N}$, and $D \subset \Omega$. The fresco problem is modeled as the reconstruction/restoration of a vector valued function $u: \Omega \rightarrow \mathbb{R}^{M}$ from a given observed couple of functions $y^{\delta}:=(\bar{u}, \bar{v})$. The observed function $\bar{u}$ is assumed to represent correct information on $\Omega \backslash D$, and $\bar{v}$ the result of a nonlinear distortion $\mathcal{L}: \mathbb{R}^{M} \rightarrow \mathbb{R}$ on $D$.

In particular, a digital image can be modeled as a function $u: \Omega \subset \mathbb{R}^{2} \rightarrow \mathbb{R}_{+}^{3}$, so that, to each "point" $\mathbf{x}$ of the image, one associates the vector $u(\mathbf{x})=(r(\mathbf{x}), g(\mathbf{x}), b(\mathbf{x})) \in \mathbb{R}_{+}^{3}$ of the color represented by the different channels red, green, and blue. In particular, a digitalization of the image $u$ corresponds to its sampling on a regular lattice $\tau \mathbb{Z}^{2}, \tau>0$. Let us again write $u: \mathcal{N} \rightarrow \mathbb{R}_{+}^{3}, u(\mathbf{x})=(r(\mathbf{x}), g(\mathbf{x}), b(\mathbf{x}))$, for $\mathbf{x} \in \mathcal{N}:=\Omega \cap \tau \mathbb{Z}^{2}$.

Usually the gray level of an image can be described as a submanifold $\mathcal{M} \subset \mathbb{R}^{3}$ by

$$
\mathcal{M}:=\mathcal{M}_{\sigma}=\left\{\sigma(x): x=\mathcal{L}(r, g, b):=L\left(\xi_{1} r+\xi_{2} g+\xi_{3} b\right),(r, g, b) \in \mathbb{R}_{+}^{3}\right\},
$$

where $\xi_{1}, \xi_{2}, \xi_{3}>0, \xi_{1}+\xi_{2}+\xi_{3}=1, L: \mathbb{R} \rightarrow \mathbb{R}$ is a non-negative increasing function, and $\sigma: \mathbb{R}_{+} \rightarrow \mathbb{R}_{+}^{3}$ is a suitable section such that $\mathcal{L} \circ \sigma=\mathrm{id}_{\mathbb{R}_{+}}$. The function $L$ is assumed smooth, nonlinear, and normally nonconvex and nonconcave. In practice, the function $L$ and thus $\mathcal{L}$ is unknown. Thus, the reconstruction of an incomplete color image has to be done in two steps: Firstly, the operator $\mathcal{L}$ has to be estimated from the given (incomplete) color data and the available noisy gray scale image $\bar{v}$, and secondly, the full color image has to be reconstructed as a solution of the equation $\mathcal{L}(u)=\bar{v}$. For example, Figure 2. describes the typical shape of an $L$ function, which is estimated by fitting a distribution of data from the real color fragments, e.g., as in Figure 1.

As first proposed in [28] we would like to minimize the discrepancy with respect to the data

$$
\Delta\left(u, \mathcal{L}, y^{\delta}\right):=\underbrace{\int_{D}|\mathcal{L}(u(x))-\bar{v}(x)|^{2} d x}_{=: G_{1}(u)}+\underbrace{\mu \int_{\Omega \backslash D}|u(x)-\bar{u}(x)|^{2} d x}_{=: G_{2}(u)},
$$

where $\mu>0$ is a positive constant which rules the mutual importance of the two terms. For example, Figure 1 illustrates a typical situation where this model applies. In fact, in this case, there is an area $\Omega \backslash D$ of the domain $\Omega \subset \mathbb{R}^{2}$ of the image, where some fragments with colors are placed and complete information is available, and an other area $D$ (which we call the inpainting region) where only the gray level information is known, modeled as the image of $\mathcal{L}$. The hope 


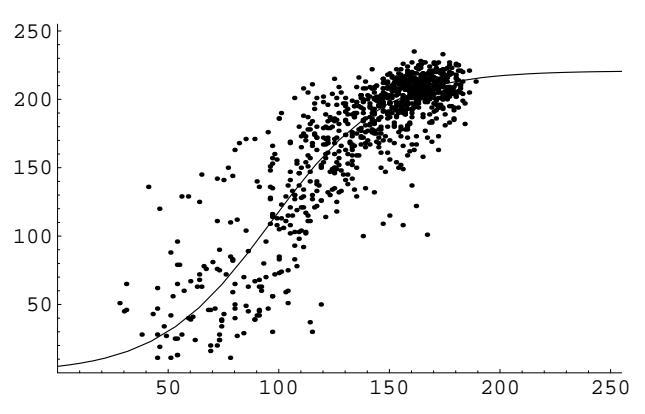

Figure 2: Estimate of the nonlinear curve $L$ from a distribution of points with coordinates given by the linear combination $\xi_{1} r+\xi_{2} g+\xi_{3} b$ of the $(r, g, b)$ color fragments (abscissa) and by the corresponding underlying gray level of the original photographs dated to 1920 (ordinate). The sensitivity parameters $\xi_{1}, \xi_{2}, \xi_{3}$ to the different frequencies of red, green, and blue are chosen in order to minimize the total variance of the ordinates.

is that by minimizing (1) we can produce a new color image that extends the colors of the fragments in the gray region. Once the extended color image is transformed by means of $\mathcal{L}$, it is constrained to match the known gray level.

Unfortunately the problem (1) is not well-posed. Of course, there exist infinite possible color solutions which match the same gray level, and thus the map $\mathcal{L}$ cannot be inverted uniquely. Moreover, we have to assume, as clearly shown in Figure 2, that the datum $y^{\delta}$ is affected by a significant and ineliminable noise. As the inversion process is also usually unstable, it is fundamental to enforce a regularization mechanism [26]. Regularization is imposed by adding prior knowledge of realistic solutions which are not furnished by the sole data. This complementary information, together with the datum, might be sufficiently characterizing the solution to make it indeed unique. The regularization methods we proposed are based on the same intuition, although they differ in terms of the specific realization: Digital images can be characterized by a very limited amount of information with respect to the dimension of the pixel space. This information is especially encoded in the morphology of the image given by its significant edges. Indeed, by assuming a simplified model of an image as a piecewise constant (color) function, it is sufficient to know where the discontinuities are and the color information of one sole pixel for each connected component in order to characterize uniquely the image. In this context we say that an image is sparse. We want to incorporate the prior information as an additional term to the discrepancy with respect to data, reducing the problem to the minimization of the following general functional:

$$
J_{\alpha, \mu}(u)=\Delta\left(u, \mathcal{L}, y^{\delta}\right)+2 \alpha \Psi(u),
$$

where $\Delta$ stands for the data misfit term involving the nonlinear relation $\mathcal{L}$ between the given partial and noisy gray/color information $y^{\delta}$, with noise level bounded by $\left\|y-y^{\delta}\right\| \leq \delta$, and the complete color image $u$ and $\Psi$ is a suitable convex function. The positive parameter $\alpha$ weights the influence of the data misfit term and the penalty functional $\Psi$. In regularization theory one aims at parameter choice rules $\alpha=\alpha(\delta)$ that ensure a convergence (for $\delta \rightarrow 0$ ) of minimizers of the functional (2) to the true solution of the problem.

The two approaches we want to present here differ mainly in the chosen penalty functional $\Psi$ and in the proposed minimization strategies for the functionals:

- In the model proposed by Teschke and Ramlau [45], the function $\Psi$ measures the sparsity of the image $u$ with respect to a frame expansion, in particular wavelet or curvelet expansions. This means that we would like to promote solutions whose expansions have only few 
nonzero coefficients. Typically $\Psi$ is a suitable weighted $\ell_{p}$ norm of the sequence of the wavelet/curvelet coefficients;

- In the model proposed by Fornasier in [28], later analyzed with March in [29], the function $\Psi$ measures the total variation of the image $u$. It is well-known that this choice tends to promote solutions which are piecewise smooth, hence with sparse gradients.

Let us notice that unified approaches to both these models for sparse solution of inverse problems appear, e.g., in [15, 18, 21, 22]. Moreover, the near-equivalence of constraints promoting sparse wavelet coefficients and BV norm was established in $[16,17]$. However, the significant perceptual differences of restored images by means of these two constraints are also well-known.

Another difference of the applications of the two methods which appear in this paper is the fact that in the first approach we analyse the minimization of the fully nonlinear problem, while in the second case, based on total variation minimization, we linearize the function $\mathcal{L}$ by means of a suitable re-equalization of the gray level. This choice is made in order to facilitate the numerical solution of the system of nonlinear PDEs which arises in the second case. For both the approaches we describe the models, the algorithms for the numerical minimization, and their convergence.

The content of the paper is as follows. In Section 2 the wavelet-based joint sparsity model is reviewed together with the formulation of an efficient iterative thresholding algorithm for its solution. In Section 3 we report the mathematical results associated to the total variation minimization model and its numerical solution. In Section 4 we show the novel numerical experiments in the real-life case and the comparison of the two methods.

\section{Wavelet based recolorization by $\ell_{p}$-joint sparsity}

A sparse representation of an element in a Hilbert space is a series expansion with respect to an orthonormal basis or a frame that has only a small number of large/nonzero coefficients. Several types of signals appearing in nature admit sparse frame expansions and thus, sparsity is a realistic assumption for a very large class of problems [36]. The recent observation that it is possible to reconstruct sparse signals from vastly incomplete information $[6,5,23]$ stimulated a new fruitful line of research which is called sparse recovery or compressed sensing. This section is devoted to the outline of the relations between sparse reconstructions, vector valued functions and their application to color images. Multi-channel signals (i.e., vector valued functions) may not only possess sparse frame expansions for each channel individually, but additionally the different channels can also exhibit common sparsity patterns. Color images are multi-channel signals, exhibiting a very rich morphology. In particular, discontinuities may appear in all the channels at the same locations. This will be reflected, e.g., in sparse wavelet/curvelet expansions $[4,24]$ with relevant coefficients appearing at the same labels, or in turn in sparse gradients with supports at the same locations. Hence, an adequate sparsity constraint is a so-called common or joint sparsity measure that promotes patterns of multi-channel data that do not belong only to one individual channel but to all of them simultaneously.

This aspect of common sparsity patterns was quite recently under consideration, e.g. in $[46,47]$. In the framework of inverse problems this issue was discussed in [31, 30]. In the latter paper the authors proposed an algorithm for solving vector valued linear inverse problems with common sparsity constraints. However, as recalled in the introduction, the conversion function $\mathcal{L}$ from colors to gray levels, which should be inverted for recoloring the images, is strongly nonlinear. Although we could linearize the problem, as we do in the following section, here we want to address also the fully nonlinear case. The theory developed in $[31,30]$ is not sufficient for this purpose and needed to be extended as proposed in [45]. In the following we revise an 
iterative thresholding scheme for solving the recolorization ill-posed problem with joint sparsity constraints.

\subsection{Wavelet frames, joint sparsity and recolorization model}

Since we aim to represent the image to be reconstructed by means of a wavelet frame, we first briefly review some basics on frames followed by the detailed formulation of the recolorization problem.

Frames. Let $\Lambda$ be a countable set of indices and $X$ a separable Hilbert space. Assume we are given a preassigned set of functions $\Psi:=\left\{\phi_{\lambda}: \lambda \in \Lambda\right\} \subset X$ The set $\Psi$ is called a frame if there exist constants $A, B$ with $0<A \leq B<\infty$ such that for all $u \in X$,

$$
A\|u\|_{X}^{2} \leq \sum_{\lambda \in \Lambda}\left|\left\langle u, \phi_{\lambda}\right\rangle\right|^{2} \leq B\|u\|_{X}^{2}
$$

Associated with a frame $\Psi$, we can consider the bounded operators

$$
F: X \rightarrow \ell_{2}(\Lambda): u \mapsto\left(\left\langle u, \phi_{\lambda}\right\rangle\right)_{\lambda \in \Lambda},
$$

called the analysis operator, and its adjoint

$$
F^{*}: \ell_{2}(\Lambda) \rightarrow X: \boldsymbol{u} \mapsto \sum_{\lambda \in \Lambda} u_{\lambda} \phi_{\lambda}
$$

called the synthesis operator.

Frames are typically 'overcomplete', i.e., for a given $u \in X$, one may find many different sequences $\boldsymbol{u} \in \ell_{2}$ such that $u=\sum_{\lambda \in \Lambda} u_{\lambda} \phi_{\lambda}$. A few of them have special properties for which they are preferred, e.g., a sequence with minimal $\ell_{p}(\Lambda)$ norm. Choosing $1 \leq p<2$ ensures the reconstruction of a sparser sequence $\boldsymbol{u}$ than the classical choice of $p=2$ which corresponds to the canonical dual coefficients defined by $\left(\left\langle u,\left(F^{*} F\right)^{-1} \phi_{\lambda}\right\rangle\right)_{\lambda \in \Lambda}$.

Joint sparsity and recolorization model. In order to cast the recolorization problem as an inverse problem leading to some variational functional of the form (2) with a sparsity constraint, we firstly have to modify the functional in order to act on frame coefficient sequences. As in the introduction we assume that $u: \Omega \subset \mathbb{R}^{2} \rightarrow \mathbb{R}_{+}^{3}$ is a color (red, green, blu) channel image. We model $u$ as a square integrable vector valued function, i.e., $u \in L_{2}(\Omega)^{3}$. We also assume that $\Psi:=\left\{\phi_{\lambda}: \lambda \in \Lambda\right\}$ is frame for $L_{2}(\Omega)$, e.g., a wavelet or curvelet frame. For each channel component of $u=\left(u^{1}, u^{2}, u^{3}\right)$ we can associate the corresponding sequence of vector frame coefficients $\boldsymbol{u}=\left(\boldsymbol{u}_{\lambda}:=\left(u_{\lambda}^{1}, u_{\lambda}^{2}, u_{\lambda}^{3}\right)\right)_{\lambda \in \Lambda}$. We also denote $\boldsymbol{u}^{\ell}=\left(u_{\lambda}^{\ell}\right)_{\lambda \in \Lambda}, \ell=1,2,3$. With a slight abuse of notation we can write

$$
u=F^{*} \boldsymbol{u}:=\left(F^{*} \boldsymbol{u}^{1}, F^{*} \boldsymbol{u}^{2}, F^{*} \boldsymbol{u}^{3}\right)=\sum_{\lambda \in \Lambda} \boldsymbol{u}_{\lambda} \phi_{\lambda} .
$$

Following the arguments in $[31,30,46]$ on joint sparsity, a reasonable measure that forces a coupling of non-vanishing color components is of the form

$$
\Psi(\boldsymbol{u})=\sum_{\lambda \in \Lambda} \omega_{\lambda}\left\|\boldsymbol{u}_{\lambda}\right\|_{q}^{p}
$$

with $q \in[1,2], p \in\{1, q\}$ and $\omega_{\lambda} \geq c>0$. This penalty term $\Psi$ is a joint sparsity measure. 
The choice of this regularization measure was encouraged by the fact that several inpainting approaches deal with total variation penalties $[12,14]$. Since in 2 dimensions, $\ell_{1}$ sparse wavelet expansions are known to give 'near' minimal total variation solutions, see, e.g., $[17,20]$, this kind of sparsity constraint seems to be well suited for our purpose. Forcing, moreover, for common sparsity patterns (e.g., edges in color images), a coupling of the different color channels is advantageous and can be achieved when setting $p=1$.

Thus the full functional for the color inpainting problem reads as follows

$$
J_{\alpha, \mu}(\boldsymbol{u}):=J_{\alpha, \mu, q}(\boldsymbol{u}):=\underbrace{\left\|\left(\mathcal{L}\left(F^{*} \boldsymbol{u}\right)-\bar{v}\right)\right\|_{L_{2}(\Omega)}^{2}}_{=G_{1}(\boldsymbol{u})}+\mu \underbrace{\left\|\left(F^{*} \boldsymbol{u}-\bar{u}\right) \chi_{D}\right\|_{\left(L_{2}(\Omega)\right)^{3}}^{2}}_{=G_{2}(\boldsymbol{u})}+2 \alpha \sum_{\lambda \in \Lambda} \omega_{\lambda}\left\|\boldsymbol{u}_{\lambda}\right\|_{q}^{p} .
$$

An approximation $u=F^{*} \boldsymbol{u}$ to the original color image is now computed by means of the minimizer $\boldsymbol{u}$ of (5). Unfortunately, a direct approach towards its minimization leads to a nonlinear optimality system where the frame coefficients are coupled. Instead, we propose to replace (5) by a sequence of functionals that are much easier to minimize and for which the sequence of the corresponding minimizers converges at least to a critical point of (5). To be explicit, for $\boldsymbol{u} \in \ell_{2}(\Lambda)^{3}$ and some auxiliary $\boldsymbol{a} \in \ell_{2}(\Lambda)^{3}$, we define a surrogate functional

$$
J_{\alpha, \mu, q}^{s}(\boldsymbol{u}, \boldsymbol{a}):=J_{\alpha, \mu, q}(\boldsymbol{u})+C\|\boldsymbol{u}-\boldsymbol{a}\|_{\ell_{2}(\Lambda)^{3}}^{2}-\left\|\mathcal{L}\left(F^{*} \boldsymbol{u}\right)-\mathcal{L}\left(F^{*} \boldsymbol{a}\right)\right\|_{L_{2}(\Omega)}^{2}
$$

and create an iteration process by:

1. Pick $\boldsymbol{u}_{0} \in \ell_{2}(\Lambda)^{3}$ and some proper constant $C>0$

2. Derive a sequence $\left(\boldsymbol{u}_{k}\right)_{k=0,1, \ldots}$ by the iteration:

$$
\boldsymbol{u}_{k+1}=\arg \min _{\boldsymbol{u} \in \ell_{2}(\Lambda)^{3}} J_{\alpha, \mu, q}^{s}\left(\boldsymbol{u}, \boldsymbol{u}_{k}\right) \quad k=0,1,2, \ldots
$$

It will turn out that the minimizers of the surrogate functionals are easily computed. In particular, the problem decouple, and every frame coefficient can be treated separately. For linear operators $\mathcal{L}$ this algorithm has been analyzed in [19]. In the nonlinear case, some weak assumptions (exhibiting no significant restriction) have to be made, e.g., on the function $\mathcal{L}$, in order to ensure existence of global minimizers, norm convergence of the iterates $\boldsymbol{u}_{k}$, and regularization properties.

\subsection{Minimization, convergence and regularization}

In this section we review the basic steps that are required for the minimization of the surrogate functional (6) and for showing norm convergence and regularization properties. For all details, including proofs, we refer to [45].

Minimization. Since the Tikhonov functional is nonconvex (because of the nonlinearity of the operator $\mathcal{L}$ ) and nondifferentiable due to the $\ell_{p}$ sparsity constraint, the proposed algorithm is based on subdifferential calculus leading to the involvement of nonlinear projections and delivering (in general) not a global but rather a local optimum of (5). To initialize the algorithm, we assume that we are given some $\alpha>0$ and some initial $\boldsymbol{u}_{0} \in \ell_{2}(\Lambda)^{3}$. An important ingredient for the the convergence of the surrogate functional approach is the choice of the constant $C$ in (6). If it is chosen too small, then the iteration blows up; chosen too big, however, the convergence of the algorithm is rather slow. A value for $C$ that ensures the convergence of the algorithm can be derived directly out of the initial iterate $\boldsymbol{u}_{0}$, see [42]. However, we wish to remark that numerical experiments suggest that $C$ is usually estimated too large, even much 
smaller values usually ensure a much faster convergence. In the following, we will always assume that $C$ was chosen appropriately.

The necessary condition for a minimum of $J_{\alpha, \mu, q}^{s}(\boldsymbol{u}, \boldsymbol{a})$ can be derived via subdifferential calculus as

$$
\left.\left.0 \in \mathcal{L}^{\prime}\left(F^{*} \boldsymbol{u}\right)^{*}\left(\bar{v}-\mathcal{L}\left(F^{*} \boldsymbol{a}\right)\right)-\mu F\left(F^{*} \boldsymbol{u}-\bar{u}\right) \chi_{D}\right) / C+\boldsymbol{a}\right) .
$$

For the case $p=1, q \geq 1$ in $\Psi$, it can be rewritten as a fixed point equation

$$
\left.\boldsymbol{u}=\frac{\alpha}{C}\left(I-P_{\mathcal{C}}\right)(\frac{C}{\alpha} \underbrace{\left(\mathcal{L}^{\prime}\left(F^{*} \boldsymbol{u}\right)^{*}\left(\bar{v}-\mathcal{L}\left(F^{*} \boldsymbol{a}\right)-\mu F\left(F^{*} \boldsymbol{u}-\bar{u}\right) \chi_{D}\right) / C+\boldsymbol{a}\right)}_{=: M(\boldsymbol{u}, \boldsymbol{a})})\right),
$$

where $P_{\mathcal{C}}$ is the orthogonal projection onto a convex set $\mathcal{C}$ satisfying $\Psi^{*}=\chi_{\mathcal{C}}$ (and $\Psi^{*}$ denoting the Fenchel transform of $\Psi$ ), see [42], Lemma 6. By our choice of $C$ it turns out that the associated fixed point map

$$
\Phi(\boldsymbol{u}, \boldsymbol{a})=\frac{\alpha}{C}\left(I-P_{\mathcal{C}}\right)\left(\frac{M(\boldsymbol{u}, \boldsymbol{a})}{\frac{\alpha}{C}}\right)
$$

is, for any generic anchor $\boldsymbol{a}=\boldsymbol{u}_{k}, k=0,1, \ldots$, a contractive operator, and thus we obtain the new iterate $\boldsymbol{u}_{k+1}$ from $\boldsymbol{u}_{k}$ by a fixed point iteration. To see this, we have to keep in mind that $I-P_{\mathcal{C}}$ is a nonexpansive operator, which reduces the task of estimating $\|\Phi(\boldsymbol{u}, \boldsymbol{a})-\Phi(\tilde{\boldsymbol{u}}, \boldsymbol{a})\|$ to the estimation of $\|M(\boldsymbol{u}, \boldsymbol{a})-M(\tilde{\boldsymbol{u}}, \boldsymbol{a})\|$, which can be done by standard methods. The computation of the fixed point iterates requires the evaluation of the operator $I-P_{\mathcal{C}}$ :

Lemma 1 Let $p=1,1 \leq q \leq \infty$ and let $l$ denote the index of the fixed point iteration. Then, the coefficients of the fixed point iterates in (8) are given by

$$
\left(\boldsymbol{u}_{l+1}\right)_{\lambda}=\left(\left(\boldsymbol{u}_{l+1}^{1}\right)_{\lambda}, \ldots,\left(\boldsymbol{u}_{l+1}^{M}\right)_{\lambda}\right)=\left(I-P_{B_{q^{\prime}}\left(C^{-1} \alpha \omega_{\lambda}\right)}\right)\left(M_{\lambda}\left(\boldsymbol{u}_{l}, \boldsymbol{a}\right)\right),
$$

where $P_{B_{q^{\prime}}\left(C^{-1} \alpha \omega_{\lambda}\right)}$ denotes the orthogonal projection onto the ball

$$
B_{q^{\prime}}\left(C^{-1} \alpha \omega_{\lambda}\right)=\left\{\boldsymbol{v} \in \mathbb{R}^{M}:\|\boldsymbol{v}\|_{q^{\prime}} \leq C^{-1} \alpha \omega_{\lambda}\right\}
$$

with $1=1 / q+1 / q^{\prime}$.

The proof of this result can be easily retraced in [45]. In general, the evaluation of $P_{B_{q^{\prime}}\left(C^{-1} \alpha \omega_{\lambda}\right)}$ is rather difficult and only for individual cases explicitly given, e.g. for $q \in\{1,2, \infty\}$ the expression can be found in $[31,30]$ (see also Section 4.1 for our specific choice). Since for $p=q$ the variational equations decouple, the necessary conditions can be evaluated directly without operating with duality arguments. The unique fixed point of $\Phi\left(\boldsymbol{u}, \boldsymbol{u}_{k}\right)$ is the unique minimizer of the surrogate functional $J_{\alpha, \mu, q}^{s}\left(\boldsymbol{u}, \boldsymbol{u}_{k}\right)$. Thus, by employing a fixed point iteration, we are able to reconstruct the new iterate $\boldsymbol{u}_{k+1}$.

Convergence. First it can be shown that there exists at least a convergent subsequence $\boldsymbol{u}_{k_{l}}$ with weak limit $\boldsymbol{u}_{\star}$, which turns out to be a critical point point for the functional $J_{\alpha, \mu}$. Using the properties of the functional $\Psi$ it can also be shown that the subsequence converges also in the $\ell_{2}(\Lambda)^{3}$-norm. However, the limits of different convergent subsequences $\left(\boldsymbol{u}_{k_{l}}\right) \subset\left(\boldsymbol{u}_{k}\right)$ may differ, but they will all have the same value of the functional $J_{\alpha, \mu}$. In the next theorem (where the first assertion can be directly taken from [41] and the second assertion is just repeating [42, Lemma 16]) we give a simple criterion that ensures strong convergence of the whole sequence $\left(\boldsymbol{u}_{k}\right)$ towards a critical point of $J_{\alpha, \mu, q}$. 
Theorem 2 Assume that there exists at least one isolated limit $\boldsymbol{u}_{\star}$ of a subsequence $\left(\boldsymbol{u}_{k_{l}}\right) \subset$ $\left(\boldsymbol{u}_{k}\right)$. Then $\boldsymbol{u}_{k} \rightarrow \boldsymbol{u}_{\star}$ in norm as $k \rightarrow \infty$. The accumulation point $\boldsymbol{u}_{\star}$ fulfills a necessary condition (i.e., the Euler-Lagrange condition) for a minimizer of $J_{\alpha, \mu, q}$.

Regularization properties. We also briefly discuss the regularization properties of the proposed iterative approach. We consider minimizers $\boldsymbol{u}_{\mu_{k} \alpha_{k}}^{\delta_{k}}$ of the functional

$$
J_{\alpha_{k}, \mu_{k}, q}(\boldsymbol{u}):=\left\|\mathcal{L}\left(F^{*} \boldsymbol{u}\right)-\bar{v}^{\delta_{k}}\right\|_{L_{2}(\Omega)}^{2}+\mu_{k} G_{2}(\boldsymbol{u})+2 \alpha_{k} \Psi(\boldsymbol{u}) .
$$

For some a-priori parameter choice rule for $\mu$ and $\alpha$ it is shown in [45] that the sequence of minimizers of $(9)$ (for $\delta \rightarrow 0$ ) contains a convergent subsequence for which the limit is a solution for $\mathcal{L}\left(F^{*} \boldsymbol{u}\right)=\bar{v}$ with minimal value of $G_{2}+\Psi$.

Theorem 3 Let $\bar{v}^{\delta_{k}} \in L_{2}(\Omega)$ be given with $\left\|\bar{v}^{\delta_{k}}-\bar{v}\right\| \leq \delta_{k}$ and $\delta_{k} \rightarrow 0$ for $k \rightarrow \infty$, and

$$
\Psi(\boldsymbol{u})=\sum_{\lambda \in \Lambda} \omega_{\lambda}\left\|\boldsymbol{u}_{\lambda}\right\|_{q}^{p}
$$

If the regularization parameters are chosen such that $\mu_{k} \rightarrow 0, \alpha_{k} \rightarrow 0, \delta_{k}^{2} / \min \left\{\mu_{k}, \alpha_{k}\right\} \rightarrow 0$ and $\max \left\{\mu_{k}, \alpha_{k}\right\} / \min \left\{\mu_{k}, \alpha_{k}\right\} \rightarrow 1$ as $k \rightarrow \infty$, then every sequence of minimizers $\boldsymbol{u}_{\alpha_{k}}^{\delta_{k}}$ of (9) has a convergent subsequence in $\ell_{2}(\Lambda)^{3}$. Every limit is a solution of the equation $\mathcal{L}\left(F^{*} \boldsymbol{u}\right)=\bar{v}$ with minimal value of $G_{2}+\Psi$. If the solution of the equation is unique, then the whole sequence converges.

This results answers the question of a faithful reconstruction at least partially. If we have reasonable data available, and the color to gray converter is known, then the reconstruction will be close to a solution of the equation $\mathcal{L}\left(F^{*} \boldsymbol{u}\right)=\bar{v}$, which means that only these colors will be exactly reconstructed where $\mathcal{L}$ is invertible.

\subsection{Numerical Implementation}

Summarizing the theoretical results in the wavelet-based approach, an approximation of the original color image is computed as a numerical approximation of a minimizer of (5), which is realized by the suggested iterative approach (7). As we have seen in formula (8), each iterate $\boldsymbol{u}_{k}$ has to be derived via a fixed point equation finally leading to the following

\section{Algorithm WAVELET-JOINT-SPARSE}

Input:

nonlinear operator $\mathcal{L}$ (estimated on the basis of the given color fragments); data $y^{\delta}=\left(\bar{u}, \bar{v}\right.$ ); initial guess $\boldsymbol{u}_{0} \in\left(\ell_{2}\right)^{n}$ (can be chosen arbitrarily, a good choice the given by the gray image); precision accuracy $\varepsilon_{\text {fixed }}>0$ for the inner fixed point iteration; maximal number $n_{\max }$ of outer iterations; proper constant $C$ (in dependence on $\boldsymbol{u}_{0} \in\left(\ell_{2}\right)^{n}$ ); weight sequence $\left\{w_{\lambda}\right\}$ (a simple choice is $w_{\lambda}=1$ for all $\lambda$ )

Parameters: parameter $\mu>0$, regularization parameter $\alpha>0, p$ and $q$ (a good choice to ensure a reasonable coupling of the three color components is $p=1, q=2$ ); wavelet (e.g. Daubechies' wavelet of order 3)

Output: numerical approximation $\tilde{\boldsymbol{u}}_{\star}$ of an accumulation point $\boldsymbol{u}_{\star}$ of (5)

Initialize $\boldsymbol{u}_{0}$

for $n=0$ to $n_{\max }-1$

\%Initialize the fixed point iteration:

$\left(\boldsymbol{u}_{n+1}\right)_{0}:=\boldsymbol{u}_{n}$

while $\operatorname{dist}\left\{\left(\boldsymbol{u}_{n+1}\right)_{l},\left(\boldsymbol{u}_{n+1}\right)_{l+1}\right\}<\varepsilon_{\text {fixed }}$ 
\%Evaluation of the fixed point map:

$\left.\left(\boldsymbol{u}_{n+1}\right)_{l+1}=\Phi\left(\left(\boldsymbol{u}_{n+1}\right)_{l}, \boldsymbol{u}_{n}\right)\right)$

end for

end while

$\boldsymbol{u}_{n+1}:=\left(\boldsymbol{u}_{n+1}\right)_{l^{*}}$

$\tilde{\boldsymbol{u}}_{\star}=\boldsymbol{u}_{n_{\max }}$

\section{Total variation minimization model and nonlinear PDEs}

Total variation minimization was first introduced in image processing for the denoising problem by the well-known Rudin-Osher-Fatemi model [43]. In several successive contributions, see, e.g., $[10,1,48]$, total variation was considered also as a regularization tool for inverse problems. Following the tracks of these fundamental contributions, we reformulated the recolorization model by choosing the function $\Psi$ as follows:

$$
\Psi(u):=\int_{\Omega} \sum_{\ell=1}^{M}\left|\nabla u^{\ell}(x)\right| d x .
$$

Note that this function measures the total variation of the vector components of the function $u=\left(u^{1}, \ldots, u^{M}\right)$. One might think that the given definition of the total variation will favor the apparition of pixels with only one pure color. The use of $\left(\sum_{\ell=1}^{M}\left|\nabla u^{\ell}(x)\right|^{2}\right)^{1 / 2}$ might avoid this problem. However, the use of a coupled TV will impose a coupling of higher order derivatives in the Euler-Lagrange equations. This is going to make the numerical implementation rather difficult. The non-coupling is however compensated by the one provided at the 0 -order terms by $\mathcal{L}$. This weaker coupling is in practice sufficient for a correct extension of the colors.

The recolorization is modeled as the minimum solution of the functional

$$
\begin{aligned}
J_{\alpha, \mu}(u) & =\Delta\left(u, \mathcal{L}, y^{\delta}\right)+2 \alpha \Psi(u) \\
& =\underbrace{\int_{D}|\mathcal{L}(u(x))-\bar{v}(x)|^{2} d x}_{=G_{1}(u)}+\mu \underbrace{\int_{\Omega \backslash D}|u(x)-\bar{u}(x)|^{2} d x}_{=G_{2}(u)}+2 \alpha \int_{\Omega} \sum_{\ell=1}^{M}\left|\nabla u^{\ell}(x)\right| d x,
\end{aligned}
$$

where we want to reconstruct the vector valued function $u:=\left(u^{1}, \ldots, u^{M}\right): \Omega \subset \mathbb{R}^{2} \rightarrow \mathbb{R}^{M}$ ( $M=3$ for RGB images) from a given observed couple of color/gray level functions $(\bar{u}, \bar{v})$. Again, $\alpha$ acts as regularization parameter, whereas $\mu$ weights the influence of the two fitting terms. For the model to make sense, in the following we assume that

(L1) $\mathcal{L}: \mathbb{R}^{M} \rightarrow \mathbb{R}_{+}$is a non-decreasing continuous function in the sense that $\mathcal{L}(x) \leq \mathcal{L}(y)$ for any $x, y \in \mathbb{R}^{M}$ such that $\left|x_{i}\right| \leq\left|y_{i}\right|$ for any $i \in\{1, \ldots, M\}$;

(L2) $\mathcal{L}(x) \leq a+b|x|^{s}$, for all $x \in \mathbb{R}^{M}$ and for fixed $s \geq 1 / 2, b>0$, and $a \geq 0$.

Moreover, one of the two following conditions holds

(L3-a) $\lim _{x \rightarrow \infty} \mathcal{L}(x)=+\infty$;

(L3-b) $\mathcal{L}(x)=\mathcal{L}\left(x_{1}, \ldots, x_{M}\right)=\mathcal{L}\left(\left(\ell_{1} \wedge x_{1} \vee-\ell_{1}\right), \ldots,\left(\ell_{M} \wedge x_{M} \vee-\ell_{M}\right)\right)$, for a suitable fixed vector $\ell=\left(\ell_{1}, \ldots, \ell_{M}\right) \in \mathbb{R}_{+}^{M}$. 
Observe that condition (L3-a) is equivalent to say that for every $C>0$ the set $\{\mathcal{L} \leq C\}$ is bounded. Therefore there exists $A \in \mathbb{R}^{M}$, with $A_{i} \geq 0$ for any $i \in\{1, \ldots, M\}$, such that $\{\mathcal{L} \leq C\} \subseteq \prod_{i=1}^{M}\left[-A_{i}, A_{i}\right]$

Under these conditions, the functional $J_{\alpha, \mu}$ is well defined in $L^{\infty}\left(\Omega ; \mathbb{R}^{M}\right) \cap W^{1,1}\left(\Omega ; \mathbb{R}^{M}\right)$. Since this space is not reflexive, and sequences that are bounded in $W^{1,1}$ are also bounded in BV (bounded variation function space), we extend $J_{\alpha, \mu}$ to the space $B V\left(\Omega ; \mathbb{R}^{M}\right)$ of vectorvalued functions in such a way that the extended functional is lower semicontinuous. By using the relaxation method of the Calculus of Variations, the natural candidate for the extended functional is the relaxed functional $\bar{J}_{\alpha, \mu}$ of $J_{\alpha, \mu}$ with respect to the componentwise BV weak*-topology. We set $X=\left\{u \in B V\left(\Omega ; \mathbb{R}^{M}\right):\left\|u_{i}\right\|_{\infty} \leq K_{i}, i=1, \ldots, M\right\}$, where, for any $i \in\{1, \ldots, M\}$, the constant $K_{i}>0$ is defined by $K_{i}=\max \left\{A_{i},\left\|\bar{u}_{i} \mid \Omega \backslash D\right\|_{\infty}\right\}$, if the condition (L3-a) holds, and by $K_{i}=\max \left\{\ell_{i},\left\|\bar{u}_{i} \mid \Omega \backslash D\right\|_{\infty}\right\}$, if the condition (L3-b) holds. The relaxed functional of $J_{\alpha, \mu}$ in $X$ with respect to the componentwise BV weak-*-topology is given by

$$
\bar{J}_{\alpha, \mu}(u)=\int_{D}|\mathcal{L}(u(x))-\bar{v}(x)|^{2} d x+\mu \int_{\Omega \backslash D}|u(x)-\bar{u}(x)|^{2} d x+\alpha \sum_{i=1}^{M}\left|D\left(u_{i}\right)\right|(\Omega),
$$

where $|D(v)|(\Omega)$ is the total variation of the BV function $v$. We have the following result.

Theorem 4 There exists a solution of the following variational problem:

$$
\min _{u \in X} \bar{J}_{\alpha, \mu}(u)
$$

In particular we have

$$
\min _{u \in X} \bar{J}_{\alpha, \mu}(u)=\inf _{u \in X} J_{\alpha, \mu}(u) .
$$

Moreover, if $D \subsetneq \Omega$ and $G_{2}$ is a strictly convex functional then the solution is unique.

For the computation of minimizers, we use a similar approach as in [10]. Let us introduce a new functional given by

$$
\mathcal{E}_{h}(u, w):=\frac{1}{\alpha}\left(G_{1}(u)+\mu G_{2}(u)\right)+\int_{\Omega} \sum_{\ell=1}^{M}\left(w^{\ell}\left|\nabla u^{\ell}(x)\right|^{2}+\frac{1}{w^{\ell}}\right) d x,
$$

where $u \in W^{1,2}\left(\Omega ; \mathbb{R}^{M}\right)$, and $w \in L^{2}\left(\Omega ; \mathbb{R}^{M}\right)$ is such that $\varepsilon_{h} \leq w^{\ell} \leq \frac{1}{\varepsilon_{h}}, \ell=1, \ldots, M, \varepsilon_{h} \rightarrow 0$ for $h \rightarrow \infty$. While the variable $u$ is again the function to be reconstructed, we call the variable $w$ the gradient weight. For any given $w^{(0)} \in L^{2}\left(\Omega ; \mathbb{R}^{M}\right.$ ) (for example $w^{(0)}:=1$ ), we define the following iterative double-minimization algorithm:

$$
\left\{\begin{array}{l}
u^{(n+1)}=\arg \min _{u \in W^{1,2}\left(\Omega ; \mathbb{R}^{M}\right)} \mathcal{E}_{h}\left(u, w^{(n)}\right) \\
w^{(n+1)}=\arg \min _{\varepsilon_{h} \leq w \leq \frac{1}{\varepsilon_{h}}} \mathcal{E}_{h}\left(u^{(n+1)}, w\right)
\end{array}\right.
$$

We have the following convergence result [29].

Theorem 5 The sequence $\left(u^{(n)}\right)_{n \in \mathbb{N}}$ has subsequences that converge strongly in $L^{2}\left(\Omega ; \mathbb{R}^{M}\right)$ and weakly in $W^{1,2}\left(\Omega ; \mathbb{R}^{M}\right)$ to a function $u_{h}^{(\infty)}$. We have that $\left(u_{h}^{(\infty)}\right)_{h}$ converges for $h \rightarrow \infty$ in $B V\left(\Omega ; \mathbb{R}^{M}\right)$ to a minimizer of $\bar{J}_{\alpha, \mu}$.

Since $\mathcal{E}_{h}(\cdot, w)$ admits minimizers, their uniqueness is equivalent to the uniqueness of the solutions of the corresponding Euler-Lagrange equations. If uniqueness of the solution is satisfied, then the algorithm (11) can be equivalently reformulated as the following two-step iterative procedure: 
- Find $u^{(n+1)}$ which solves

$$
\begin{gathered}
\int_{\Omega}\left(w_{i}^{(n)}(x) \nabla u_{i}^{(n+1)}(x) \cdot \nabla \varphi_{i}(x)+\frac{\mu}{\alpha}\left(u_{i}^{(n+1)}(x)-\bar{u}_{i}(x)\right) 1_{\Omega \backslash D}(x)\right. \\
\left.+\quad \frac{1}{\alpha}\left(\mathcal{L}\left(u^{(n+1)}(x)\right)-\bar{v}(x)\right) \frac{\partial \mathcal{L}}{\partial u_{i}}\left(u^{(n+1)}(x)\right) 1_{D}(x)\right) \varphi_{i}(x) d x=0
\end{gathered}
$$

for $i=1, \ldots, M$, for all $\varphi \in W^{1,2}\left(\Omega ; \mathbb{R}^{M}\right)$.

- Compute directly $w^{(n+1)}$ by

$$
w_{i}^{(n+1)}=\varepsilon_{h} \vee \frac{1}{\left|\nabla u_{i}^{(n+1)}\right|} \wedge \frac{1}{\varepsilon_{h}}, \quad i=1, \ldots, M .
$$

There are cases for which one can ensure uniqueness of solutions:

1. If $G_{2}$ is strictly convex then the minimizers are unique as well as the solutions of the equations.

2. In the following section we illustrate the finite element approximation of the EulerLagrange equations. Since we are interested in color image applications, we restrict the numerical experiments to the simplified case $\mathcal{L}\left(u_{1}, u_{2}, u_{3}\right)=\frac{1}{3}\left(u_{1}+u_{2}+u_{3}\right)$, i.e. we set $\xi_{i}=1 / 3$ for all $i$ and $L(x)=x$. This choice of $\mathcal{L}$ is the result of a re-equalization of the gray-level, and the numerical results confirm that again it provides a reasonable model to the problem. The main advantage of this choice of linear $\mathcal{L}$ is that it requires to solve a linear system for each iteration step in Algorithm 6, whereas a nonlinear $\mathcal{L}$ leads to a nonlinear system. Defining $\mathcal{L}$ as above, the linear systems arising from the finite element discretization are uniquely solvable for a rather large set of possible parameters $\alpha, \mu$.

\subsection{Numerical implementation}

In this section we want to present the numerical implementation of the iterative double-minimization algorithm (11) for color image restoration. As the second step of the scheme (which amounts in the up-date of the gradient weight) can be explicitly done once $u^{(n+1)}$ is computed, we are left essentially to provide a numerical implementation of the first step, i.e., the solution of the Euler-Lagrange equations.

For the solution we use a finite element approximation. We illustrate the implementation with the concrete aim of the reconstruction of a digital color image supported in $\Omega=[0,1]^{2}$ from few color fragments supported in $\Omega \backslash D$ and the gray level information where colors are missing. By the nature of this problem, we can choose a regular triangulation $\mathcal{T}$ of the domain $\Omega$ with nodes distributed on a regular grid $\mathcal{N}:=\tau \mathbb{Z}^{2} \cap \Omega$, corresponding to the pixels of the image. Associated to $\mathcal{T}$ we fix the following finite element spaces:

$$
\begin{aligned}
& \mathcal{U}=\left\{u \in C^{0}(\Omega): u \mid T \in \mathbb{P}^{1}, T \in \mathcal{T}\right\}, \\
& \mathcal{V}=\left\{w \in L^{2}(\Omega): w \mid T \in \mathbb{P}^{0}, T \in \mathcal{T}\right\},
\end{aligned}
$$

where $\mathbb{P}^{k}$ denotes the space polynomials of degree $k$. The space $\mathcal{U}$ induces the finite element space of color images given by

$$
U:=\left\{u \in W^{1,2}\left(\Omega, \mathbb{R}^{3}\right): u_{i} \in \mathcal{U}, i=1,2,3\right\} .
$$

The space $\mathcal{V}$ induces the finite element space of gradient weights given by

$$
V:=\left\{w \in L^{2}\left(\Omega, \mathbb{R}^{3}\right): w_{i} \in \mathcal{V}, i=1,2,3\right\}
$$


In order to simplify the solution of the equation and avoid the nonlinearity in the coupled terms of order 0 , we can restrict our functional to the case $\mathcal{L}\left(u_{1}, u_{2}, u_{3}\right)=\frac{1}{3}\left(u_{1}+u_{2}+u_{3}\right)$. Indeed, usually in practice the functions $L$ which define $\mathcal{L}$ are increasing functions which can be inverted. Therefore we can always re-equalize the gray level in order to have the linear dependence.

For a given $w^{(n)} \in V$, the first step of our approximation of the double-minimization scheme amounts in the computation of $u^{(n+1)} \in U$ which solves

$$
\begin{gathered}
\int_{\Omega} w_{i}^{(n)}(x) \nabla u_{i}^{(n+1)}(x) \cdot \nabla \varphi_{i}(x)+\left(\frac{\mu}{\alpha}\left(u_{i}^{(n+1)}(x)-\bar{u}_{i}(x)\right) 1_{\Omega \backslash D}(x)\right. \\
\left.+\frac{1}{3 \alpha}\left(\frac{1}{3}\left(u_{1}^{(n+1)}(x)+u_{2}^{(n+1)}(x)+u_{3}^{(n+1)}(x)\right)-\bar{v}(x)\right) 1_{D}(x)\right) \varphi_{i}(x) d x=0
\end{gathered}
$$

for $i=1,2,3$, for all $\varphi \in U$. To the spaces $\mathcal{U}$ and $\mathcal{V}$ are attached the corresponding nodal bases $\left\{\varphi_{k}\right\}_{k \in \mathcal{N}}$ and $\left\{\chi_{k}\right\}_{k \in \mathcal{N}}$ respectively. Therefore, we have also that

$$
U=\left\{u: u=\left(\sum_{k \in \mathcal{N}} u_{i, k} \varphi_{k}\right)_{i=1,2,3}\right\}, \quad V=\left\{w: w=\left(\sum_{k \in \mathcal{N}} w_{i, k} \chi_{k}\right)_{i=1,2,3}\right\} .
$$

With these bases we can construct the following matrices:

$$
\begin{aligned}
\mathbf{K}_{i}^{(n+1)} & :=\left(\int_{\Omega} w_{i}^{(n)}(x) \nabla \varphi_{k}(x) \cdot \nabla \varphi_{h}(x) d x\right)_{k, h \in \mathcal{N}}, \\
\mathbf{M}_{\Omega \backslash D} & :=\left(\frac{\mu}{\alpha} \int_{\Omega} 1_{\Omega \backslash D}(x) \varphi_{k}(x) \varphi_{h}(x) d x\right)_{k, h \in \mathcal{N}}, \\
\mathbf{M}_{D} & :=\left(\frac{1}{9 \alpha} \int_{\Omega} 1_{D}(x) \varphi_{k}(x) \varphi_{h}(x) d x\right)_{k, h \in \mathcal{N}} .
\end{aligned}
$$

By these building blocks, we can assemble

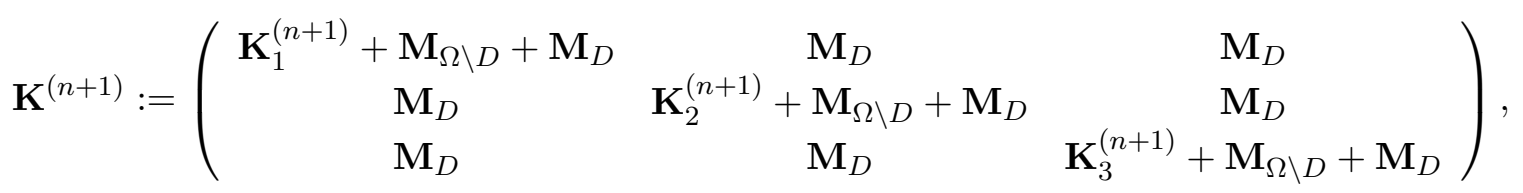

and

$$
\mathbf{M}:=\left(\begin{array}{ccc}
\mathbf{M}_{\Omega \backslash D}+\mathbf{M}_{D} & \mathbf{M}_{D} & \mathbf{M}_{D} \\
\mathbf{M}_{D} & \mathbf{M}_{\Omega \backslash D}+\mathbf{M}_{D} & \mathbf{M}_{D} \\
\mathbf{M}_{D} & \mathbf{M}_{D} & \mathbf{M}_{\Omega \backslash D}+\mathbf{M}_{D}
\end{array}\right) .
$$

Furthermore, let us denote the vector of the nodal values of the solution by

$$
\mathbf{u}^{(n+1)}=\left(u_{1, k_{1}}^{(n+1)}, \ldots, u_{1, k_{\# \mathcal{N}}}^{(n+1)}, u_{2, k_{1}}^{(n+1)}, \ldots, u_{2, k_{\# \mathcal{N}}}^{(n+1)}, u_{3, k_{1}}^{(n+1)}, \ldots, u_{3, k_{\# \mathcal{N}}}^{(n+1)}\right)^{T}
$$

assembled as a column vector containing the nodal values of each channel in order, where $k_{i} \in \mathcal{N}$ are nodes which are suitably ordered. In a similar way the nodal values of the data $\bar{u}, \bar{v}$ are assembled in the vector

$\overline{\mathbf{u}}=\left(\bar{u}_{1, k_{1}}, \ldots, \bar{u}_{1, k_{j}}, \bar{v}_{1, k_{j+1}}, \ldots, \bar{v}_{1, k_{\# \mathcal{N}}}, \bar{u}_{2, k_{1}}, \ldots, \bar{u}_{2, k_{j}}, \bar{v}_{2, k_{j+1}}, \ldots, \bar{v}_{2, k_{\# \mathcal{N}}}, \bar{u}_{3, k_{1}}, \ldots, \bar{u}_{3, k_{j}}, \bar{v}_{3, k_{j+1}}, \ldots, \bar{v}_{3, k_{\# \mathcal{N}}}\right)^{T}$

For the right-hand side we have the additional requirement that $\bar{v}_{i, k}=\bar{v}_{\ell, k}$ for $i \neq \ell$, representing the gray level values. Moreover, the order of the nodes $\left\{k_{l}: l=1, \ldots, \# \mathcal{N}\right\}$ is such that

$$
\left(\mathbf{M}_{\Omega \backslash D}+\mathbf{M}_{D}\right)\left(\bar{u}_{i, k_{1}}, \ldots, \bar{u}_{i, k_{j}}, \bar{v}_{i, k_{j+1}}, \ldots, \bar{v}_{i, k_{\# \mathcal{N}}}\right)^{T}=\mathbf{M}_{\Omega \backslash D}\left(\begin{array}{c}
\overline{\mathbf{u}}_{\mathbf{i}} \\
0
\end{array}\right)+\mathbf{M}_{D}\left(\begin{array}{c}
0 \\
\overline{\mathbf{v}}_{\mathbf{i}}
\end{array}\right) .
$$


With these notations and conventions, the solution of the system of equations (12) is equivalent to the solution of the following algebraic linear system

$$
\mathbf{K}^{(n+1)} \mathbf{u}^{(n+1)}=\mathbf{M} \overline{\mathbf{u}} .
$$

\subsection{Numerical implementation of the double-minimization algorithm}

We have now all the ingredients to assemble our numerical scheme into the following algorithm.

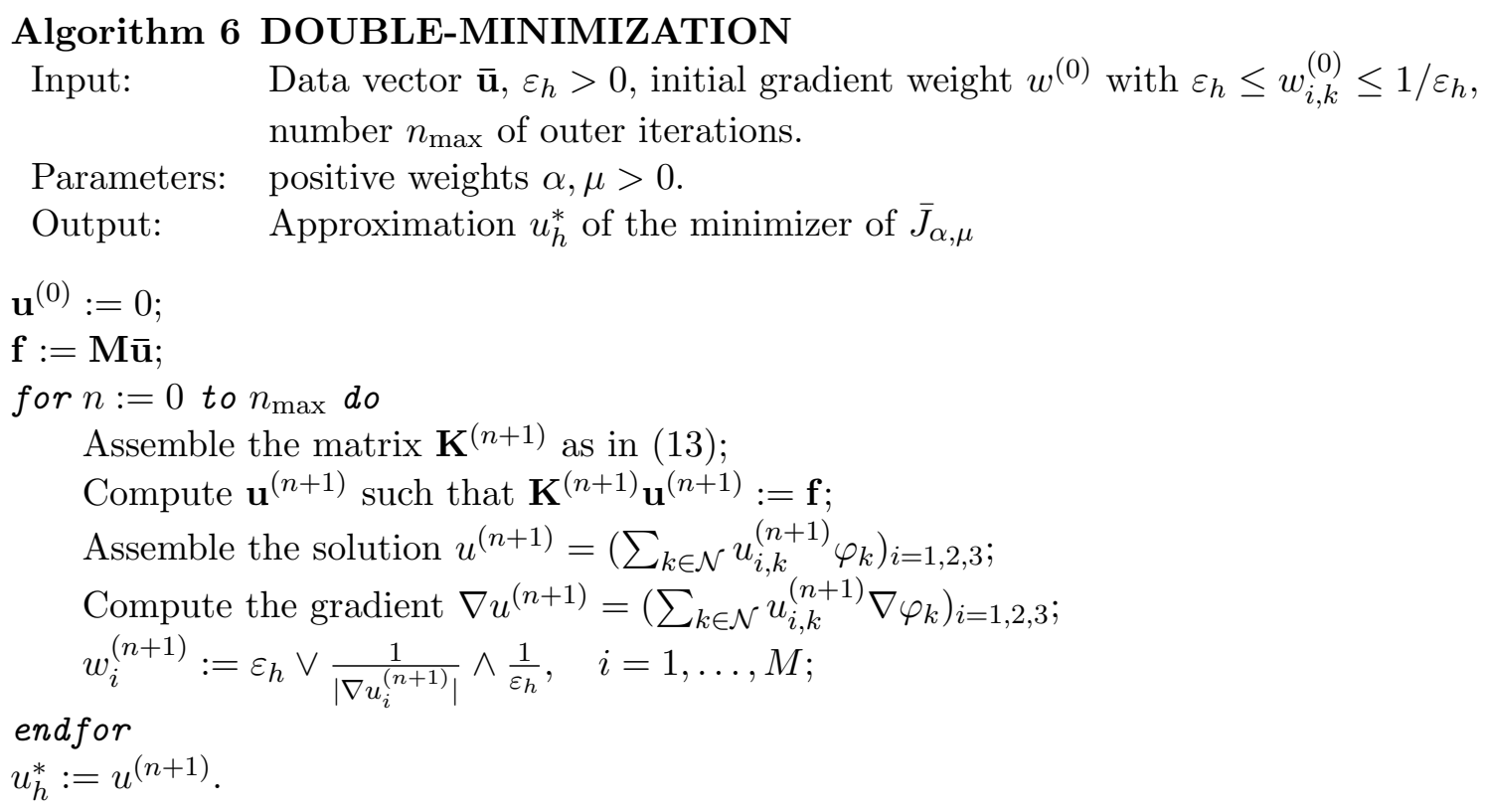

\section{Comparison of the methods in the real-life problem}

In this section, we show the numerical results that we obtained with the two methods. We assume as in Figure 1 to have available few color fragments of the image and the gray levels of the missing parts. In order to guarantee a reasonable comparison of methods, not only the data basis is required to be the same, but also the estimated color-to-gray conversion map $\mathcal{L}$ (which is nonlinear). In the wavelet approach, this map with its full nonlinearity is implemented whereas in the total variation approach a "linearization" of this map is used, by inverting the function $L$. In Figures 3 and 4 we show the estimated curves $L$ computed by suitable averaging of the color information provided by the localized fragments and from the corresponding underlying gray levels. By inverting these increasing maps we can re-equalize the gray level (as shown in the bottom-right of Figures 3 and 4) in order to obtain the desired linearization.

\subsection{Numerical experiments for the Wavelet based recolorization}

In order to derive recolored images, we first have to define the setup of the WAVELET-JOINTSPARSE algorithm. After generating our wavelet frame by Daubechies' wavelet of order 3, the initial wavelet frame coefficient vector $\boldsymbol{u}_{0}$ is derived just by applying the frame operator $F$ to each of the channels of the given gray value image. The precision accuracy $\varepsilon_{\text {fixed }}$ for the fixed point iteration is chosen to be 0.00001 (since for a contraction factor less or equal than 0.5 , the fixed point iteration converges rather quickly - typically 5-10 iterations are enough to reach the preassigned accuracy). As a reasonable value for the number of maximal outer iterations we have chosen $n_{\max }=200$. For our estimated map $\mathcal{L}$ and the chosen initial guess $\boldsymbol{u}_{0}$, our theory allows to pick $C=0.8$. For a quickly converging fixed point iteration a large value of $C$ would 

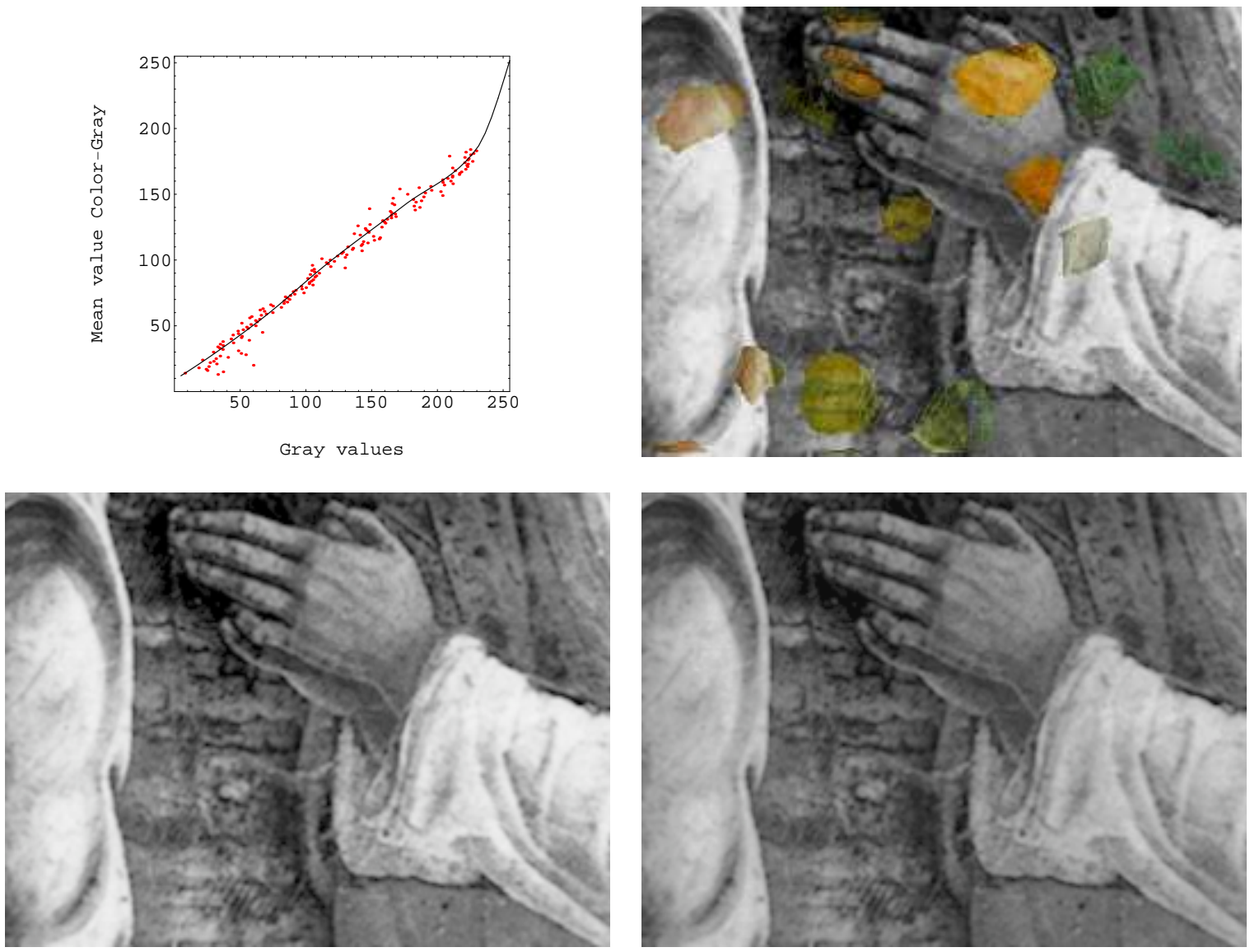

Figure 3: On the top-left we show the estimated curve $L$ on the basis of the information provided by the true colors of the localized fragments and the corresponding gray levels of the image of the fresco prior to the damage. The fragment relocated in their original position are show on the top-right figure, while the original gray level is shown on the bottom-left. The re-equalized gray level in shown on the bottom-right.

be preferable, but this on the contrary reduces the speed of convergence of the outer iteration. Therefore, we made in advance a few experiments with different values of $C$. The penalization parameter $\mu$ and the regularization parameter $\alpha$ were experimentally chosen - we end up with $\mu=0.1$ and $\alpha=0.0099$. The coupling was ensured by picking $p=1$ and $q=2$. In this situation the projection $P_{B_{2}\left(C^{-1} \alpha \omega\right)}$ (acting on the 3 channels) on the $\ell_{2}$-ball is explicitly given by

$$
P_{B_{2}\left(C^{-1} \alpha \omega\right)}\left(\boldsymbol{u}_{\lambda}\right)= \begin{cases}\boldsymbol{u}_{\lambda} & \text { if }\left\|\boldsymbol{u}_{\lambda}\right\|_{2}=\sqrt{\sum_{i=1}^{3}\left|\left(\boldsymbol{u}^{i}\right)_{\lambda}\right|^{2}} \leq C^{-1} \alpha \omega_{\lambda} \\ \frac{C^{-1} \alpha \omega_{\lambda}}{\left\|\boldsymbol{u}_{\lambda}\right\|_{2}} \boldsymbol{u}_{\lambda} & \text { otherwise }\end{cases}
$$

The results can be seen in on the bottom-left of Figures 5 and 6 .

\subsection{Numerical experiments for the total variation minimization based recol- orization}

The support of the image is $\Omega=[0,1]^{2}$ where we construct a grid of dimensions $h \times w, w$ and $h$ are the width and hight of the image in pixels respectively. On this grid a regular triangulation is defined. The values of the images are in $[0,255]$ channelwise. The algorithm converges to 
a stationary situation in a limited number of external iterations. In our numerical tests 3-4 iterations are sufficient. However the linear system $\mathbf{K}^{(n+1)} \mathbf{u}^{(n+1)}:=\mathbf{f}$ to be solved at each iteration is usually non-symmetric and hence we needed to implement BiCGStab. The choice of $\varepsilon_{h}$ has a twofold function. It serves as a regularization parameter, i.e., the visual smoothness of the reconstruction depends on $\varepsilon_{h}$. The larger values of $\varepsilon_{h}$ give smoother reconstructed images. This effect is due to the fact that if $\varepsilon_{h}$ gets large, then the corresponding differential operator becomes more and more isotropic. Moreover, since in discrete images the gradients are always bounded, if $\varepsilon_{h}$ is smaller than a threshold $T>0$ depending on the mesh size $\tau$ - in our experiments $T=(255 \max \{\text { height, width }\})^{-1}$ - then the lower bound on the gradient weight becomes irrelevant in the algorithm. However, the second purpose of this parameter is also for the sake of numerical stability. Depending on the size of the image, this parameter cannot be too small (i.e., minimal), otherwise the corresponding stiffness matrices $\mathbf{K}^{(n)}$ might be significantly ill-conditioned and suitable pre-conditioners should be invoked in this case. Indeed, it can be observed a significant increase of number of iterations in BiCGStab in order to get to an acceptable accuracy. The results in the real-life cases are shown on the bottom-right of Figures 5 and 6 .

\subsection{Comparison and conclusion}

The recolorized images due to the two methods may differ in certain aspects. The numerical results suggest that the total variation minimization method tends to preserve the edges better. At the same time it does not annihilate the information provided by the real fragments which are not completely merged with the final color restoration. On contrary, the wavelet based recolorization tends to blur more the image (which can be partly explained by the smoothness of the chosen wavelet), edges are less preserved, but the fragments are nicely merged in the context of the resulting image. These differences can be simply attributed just due a stronger regularization due to the choice of the regularization in the experiments with the wavelet based method. However, it is very difficult to tune the regularization parameters in such a way to obtain the same results, being the methods significantly different in nature.

In order to identify reconstruction differences of the two schemes, several measures seem to be appropriate. The first natural measures is the classical Euclidean distance of the individual color channels. However, the RGB channel representation of color images is not best suited in human perception of color images. A more adequate color space to represent images and to identify differences that meet human perception is the $(L, a, b)$ representation. The coordinates $L, a, b$ are based on psychological characteristics of the human perception and rely only on physical color metrics. The $L$ axis describes the luminance, the $a$ axis the green red contingent, and the $b$ axis the blue yellow contingent. The conversion from RGB to $(L, a, b)$ can be realized in two steps. The first step is the mapping from RGB to XYZ (which is the former CIE color model):

$$
\left(\begin{array}{l}
X \\
Y \\
Z
\end{array}\right)=\left(\begin{array}{ccc}
2.364 & -0.515 & 0.005 \\
-0.896 & 1.426 & -0.014 \\
-0.468 & 0.088 & 1.009
\end{array}\right)\left(\begin{array}{c}
R \\
G \\
B
\end{array}\right) .
$$

The luminance, the green-red axis, and the yellow-blue axis are then given by

$$
L=116\left(Y / Y_{n}\right)^{1 / 3}-16, \quad a=500\left(\left(X / X_{n}\right)^{1 / 3}-\left(Y / Y_{n}\right)^{1 / 3}\right), \quad b=200\left(\left(Y / Y_{n}\right)^{1 / 3}-\left(Z / Z_{n}\right)^{1 / 3}\right),
$$

where $\left(X_{n}, Y_{n}, Z_{n}\right)$ is the location of the white point. For small values of $X / X_{n}, Y / Y_{n}, Z / Z_{n}$ the third roots are replaced.

The differences in terms of edge reproduction are particularly visible in the RGB difference images, as the top rows in Figures 7 and 9 show. Interestingly, those differences are not that 
visible in the $(L, a, b)$ perceptual representation, see the bottom rows in Figures 7 and 9 . The essential differences can only be seen at the location of the fragments. This suggests that from the point of view of human perception, the reconstruction are qualitatively very close each other, at least in off-color-fragment regions.

We finally report the mutual Peak Signal-to-Noise Ratio (PSNR) ${ }^{1}$. In the first case (compare Figure 5) the PSNR between the resulting images is $30.166 \mathrm{~dB}$ and for the second case (compare Figure 6) is $29.4167 \mathrm{~dB}$.

These latter observations suggest that, if we ignore the differences due to possible edge blurring, the reconstructed color is essentially the same. Due to the significant differences of the proposed methods in terms of extension of the colors (the first is based on wavelet sparsity, the second on non-isotropic diffusion), we believe that this near equivalence is an indirect indicator of the fact that any possible recolorization method will give in practice very similar results to the one presented in this paper. The results depend on the quantity of color information and, in a very significant way, on its distribution. Previous results in $[29,45]$ showed that even a minimal color information randomly distributed is sufficient in order to achieve reconstructions with PSNR over $35 \mathrm{~dB}$.

\section{Acknowledgement}

Gerd Teschke gratefully acknowledges the partial support by Deutsche Forschungsgemeinschaft Grants TE 354/1-2, TE 354/3-1, TE 354/4-1, TE 354/5-1. Massimo Fornasier acknowledges the financial support provided by the European Union's Human Potential Programme under contract MOIF-CT-2006-039438. The paper also contributes to the Fornasier's project WWTF Five senses-Call 2006, Mathematical Methods for Image Analysis and Processing in the Visual Arts. He thanks Rocco Cazzato for the efficient implementation of Algorithm 6 [8], Domenico Toniolo and the colleagues of the Laboratory of the Mantegna Project at the University of Padova for the nice joint-work on the fragment recollocation. The work of Ronny Ramlau was supported by the Austrian Science Fund, Projects FWF- P19029, FWF-P19496-N18 and FWF-P20237-N14.

\section{References}

[1] G. Aubert and L. Vese. A variational method in image recovery. SIAM J. Numer. Anal., 34(5):1948-1979, 1997.

[2] C. Ballester, M. Beltramio, V. Caselles, G. Sapiro, and J. Verdera. Filling-in by joint interpolation of vector fields and gray levels. IEEE Trans. Image Process., 10(8):12001211, 2001.

[3] M. Beltramio, G. Sapiro, V. Caselles, and B. Ballester. Image inpainting. In SIGGRAPH 2000, July, 2001.

[4] E.J. Candès and D.L. Donoho. New tight frames of curvelets and optimal representations of objects with piecewise $C^{2}$ singularities. Commun. Pure Appl. Math., 57(2):219-266, 2004.

[5] E.J. Candès, J. Romberg, and T. Tao. Exact signal reconstruction from highly incomplete frequency information. IEEE Trans. Inf. Theory, 52(2):489-509, 2006.

[6] E.J. Candès and T. Tao. Near Optimal Signal Recovery From Random Projections And Universal Encoding Strategies. IEEE Trans. Inf. Theory, to appear.

${ }^{1} \operatorname{PSNR}\left(u^{1}, u^{2}\right):=10 \log _{10}\left(\frac{255^{2}}{\frac{1}{3 h w} \sum_{i=1}^{3}\left\|u_{i}^{1}-u_{i}^{2}\right\|_{2}^{2}}\right)$, where $h, w$ are the hight and width of the images respectively. 
[7] V. Caselles, V. Coll, and V. J.-M. Morel. Geometry and color in natural images. Journal of Mathematical Imaging and Vision, 16(2):89-105, 2002.

[8] R. Cazzato. Un Metodo per la Ricolorazione di Immagini e Altri Strumenti per il Restauro. Il Progetto Mantegna e gli Affreschi nella Chiesa degli Eremitani. Master thesis (italian), University of Padova, 2007.

[9] R. Cazzato, G. Costa, A. Dal Farra, M. Fornasier, D. Toniolo, D. Tosato, and C. Zanuso. Il Progetto Mantegna: storia e risultati. In Domenico Toniolo Anna Maria Spiazzi, Alberta De Nicolò Salmazo, editor, Andrea Mantegna e i Maestri della Cappella Ovetari: La Ricomposizione Virtuale e il Restauro, pages 151-169. Skira, 2006.

[10] A. Chambolle and P.-L. Lions. Image recovery via total variation minimization and related problems. Numer. Math., 76(2):167-188, 1997.

[11] T. F. Chan, S. H. Kang, and J. Shen. Euler's elastica and curvaure-based inpainting. SIAM J. Appl. Math., 63(2):564-592, 2002.

[12] T. F. Chan and J. Shen. Inpainting based on nonlinear transport and diffusion. Contemp. Math., 313:53-65, 2002.

[13] T. F. Chan and J. Shen. Mathematical models for local nontexture inpaintings. SIAM J. Appl. Math., 62(3):1019-1043, 2002.

[14] T. F. Chan and J. Shen. Variational image inpainting. Commun. Pure Appl. Math., 58(5):579-619, 2005.

[15] C. Chaux, P. L. Combettes, J.-C. Pesquet, and V. R. Wajs. A variational formulation for frame-based inverse problems. Inverse Probl., 23(4):1495-1518, 2007.

[16] A. Cohen, W. Dahmen, I. Daubechies, and R. DeVore. Harmonic analysis of the space BV. Rev. Mat. Iberoam., 19(1):235-263, 2003.

[17] A. Cohen, R. DeVore, P. Petrushev, and H. Xu. Nonlinear Approximation and the Space $B V\left(\mathbb{R}^{2}\right)$. American Journal of Mathematics, (121):587-628, 1999.

[18] P. L. Combettes and V. R. Wajs. Signal recovery by proximal forward-backward splitting. Multiscale Model. Simul., 4(4):1168-1200, 2005.

[19] I. Daubechies, M. Defrise, and C. DeMol. An iterative thresholding algorithm for linear inverse problems with a sparsity constraint. Comm. Pure Appl. Math, 57:1413-1541, 2004.

[20] I. Daubechies and G. Teschke. Variational image restoration by means of wavelets: simultaneous decomposition, deblurring and denoising. Applied and Computational Harmonic Analysis, 19(1):1-16, 2005.

[21] I. Daubechies, G. Teschke, and L. Vese. Iteratively solving linear inverse problems under general convex constraints. Inverse Probl. Imaging, 1(1):29-46, 2007.

[22] I. Daubechies, G. Teschke, and L. Vese. On some iterative concepts for image restoration. In Peter W. Hawkes, editor, Advances in Imaging and Electron Physics, number 150, pages 2-52. Elsevier, 2008.

[23] D.L. Donoho. Compressed Sensing. IEEE Trans. Inf. Theory, 52(4):1289-1306, 2006. 
[24] M. Elad, J.-L. Starck, P. Querre, and D.L. Donoho. Simultaneous cartoon and texture image inpainting using morphological component analysis (MCA). Appl. Comput. Harmon. Anal., 19:340-358, 2005.

[25] M. Elad, P. Milanfar, and R. Rubinstein. Analysis Versus Synthesis in Signal Priors. Inverse Problems, 23(3):947-968,2007.

[26] H.W. Engl, M. Hanke, and A. Neubauer. Regularization of inverse problems. Mathematics and its Applications (Dordrecht). 375. Dordrecht: Kluwer Academic Publishers., 1996.

[27] M.J. Fadili, J.-L. Starck, F. Murtagh. Inpainting and Zooming using Sparse Representations. The Computer Journal, 2007.

[28] M. Fornasier. Nonlinear projection recovery in digital inpainting for color image restoration. J. Math. Imaging Vis., 24(3):359-373, 2006.

[29] M. Fornasier and R. March. Restoration of color images by vector valued BV functions and variational calculus. SIAM J. Appl. Math., 68(2):437-460, 2007.

[30] M. Fornasier and H. Rauhut. Iterative thresholding algorithms. Appl. Comput. Harmon. Anal., 2008. doi:10.1016/j.acha.2007.10.005, to appear.

[31] M. Fornasier and H. Rauhut. Recovery algorithms for vector valued data with joint sparsity constraints. SIAM J. Numer. Anal., 46(2):577-613, 2008.

[32] M. Fornasier and D. Toniolo. Computer-based recomposition of the frescoes in the Ovetari Chapel in the Church of the Eremitani in Padua. Methodology and initial results, (English/Italian). in "Mantegna nella chiesa degli Eremitani a Padova. Il recupero possibile", Ed. Skira, 2003.

[33] M. Fornasier and D. Toniolo. Fast, robust, and efficient 2D pattern recognition for reassembing fragmented images. Pattern Recognition, 38(11):2074-2087, 2005.

[34] S. H. Kang and R. March. Variational models for image colorization via Chromaticity and Brightness decomposition. IEEE Trans. Image Proc., 2006. to appear.

[35] A. Levin, D. Lischinski, and Y. Weiss. Colorization using optimization. In $A C M S I G$ GRAPH 2004, pages $689-694,2004$.

[36] S. Mallat. A Wavelet Tour of Signal Processing. Academic Press, 1998.

[37] J. Mairal, M. Elad, and G. Sapiro. Sparse Representation for Color Image Restoration. IEEE Trans. on Image Processing, 17(1):53-69, 2008.

[38] S. Masnou. Disocclusion: a variational approach using level lines. IEEE Trans. on Image Processing, 11(2):68-76, 1998.

[39] S. Masnou and J.-M. Morel. Level lines based disocclusion. In Proceedings of 5th IEEE IEEE Intl Conf. on Image Process., Chicago, pages 259-263, 1998.

[40] D. Mumford. Elastica and computer vision. In C. Bajaj, editor, Algebraic geometry and applications, pages 491-506. Springer-Verlag, 1994.

[41] R. Ramlau and G. Teschke. Tikhonov Replacement Functionals for Iteratively Solving Nonlinear Operator Equations. Inverse Problems, 21:1571-1592, 2005. 
[42] R. Ramlau and G. Teschke. A Projection Iteration for Nonlinear Operator Equations with Sparsity Constraints. Numerische Mathematik, 104:177-203, 2006.

[43] L. I. Rudin, S. Osher, and E. Fatemi. Nonlinear total variation based noise removal algorithms. Physica D, 60(1-4):259-268, 1992.

[44] G. Sapiro. Inpainting the colors. In ICIP 2005. IEEE International Conference on Image Processing, pages 698-701, 2005.

[45] G. Teschke and R. Ramlau. An iterative algorithm for nonlinear inverse problems with joint sparsity constraints in vector valued regimes and an application to color image inpainting. Inverse Problems, 23:1851-1870, 2007.

[46] J. Tropp. Algorithms for simultaneous sparse approximation. part ii: Convex relaxation. Signal Processing, special issue Sparse approximations in signal and image processing, 86:589-602, 2006.

[47] J. Tropp, A. C. Gilbert, and M. J. Strauss. Algorithms for simultaneous sparse approximation. part i: Greedy pursuit. Signal Processing, special issue Sparse approximations in signal and image processing, 86:572-588, 2006.

[48] L. Vese. A study in the BV space of a denoising-deblurring variational problem. Appl. Math. Optim., 44:131-161, 2001.

[49] L. Yatziv and G. Sapiro. Fast image and video colorization using chrominance blending. IEEE Transactions on Image Processing, 15(5):1120-1129, 2006. 

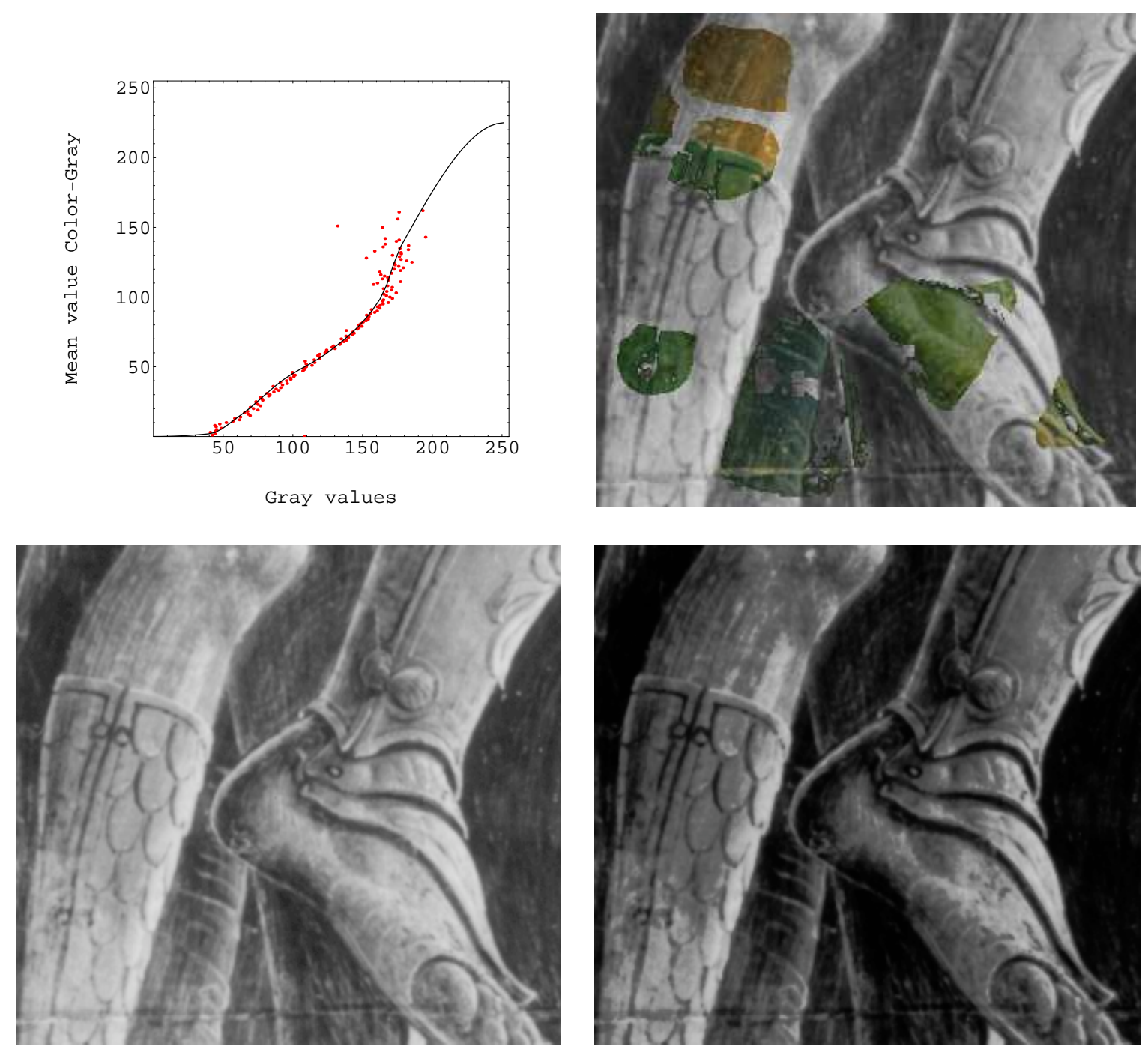

Figure 4: On the top-left we show the estimated curve $L$ on the basis of the information provided by the true colors of the localized fragments and the corresponding gray levels of the image of the fresco prior to the damage. The fragment relocated in their original position are show on the top-right figure, while the original gray level is shown on the bottom-left. The re-equalized gray level in shown on the bottom-right. 

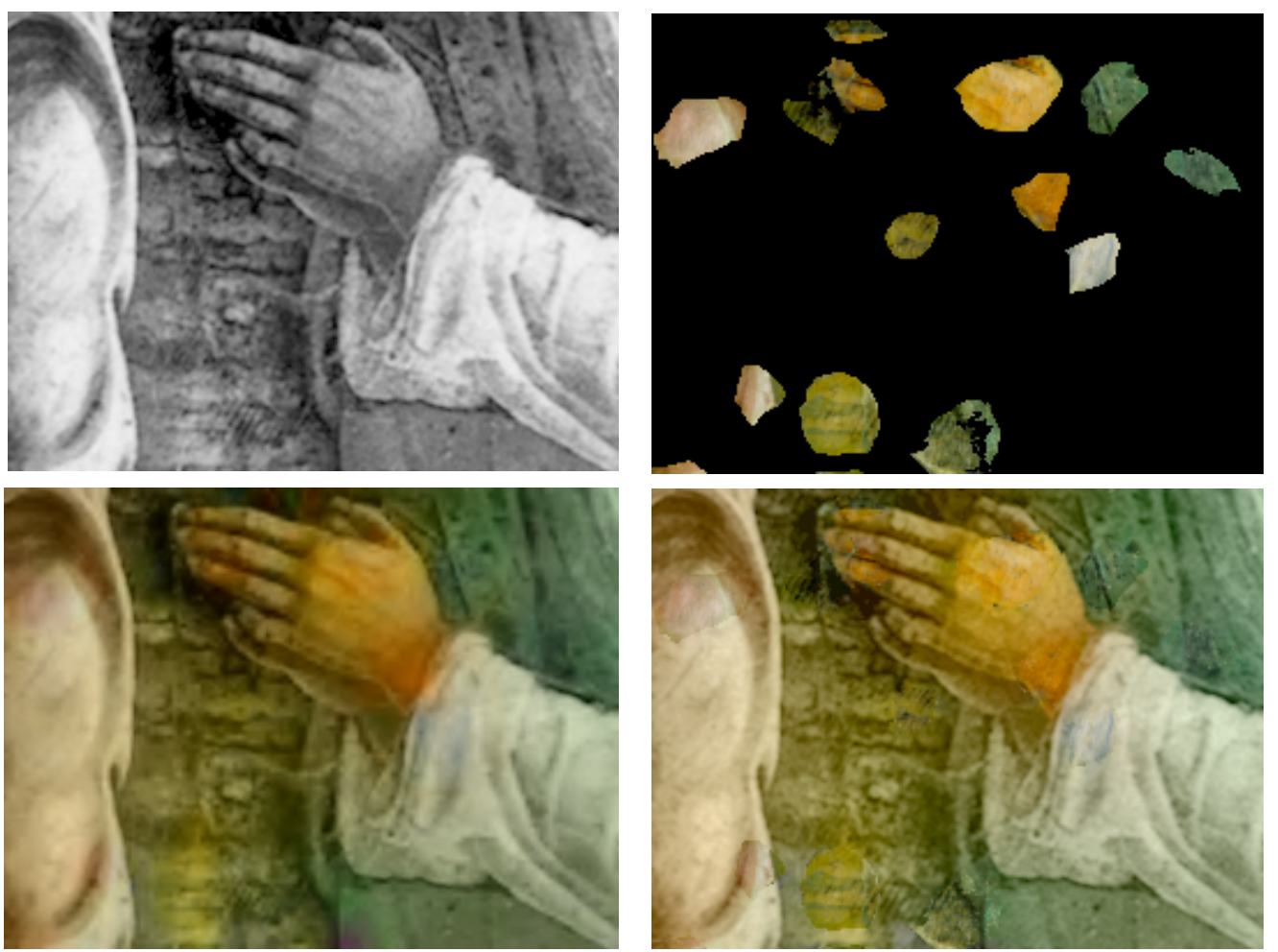

Figure 5: On the top of the figure we show the data, i.e., the gray level and the colored fragments. On the bottom-left we show the result of the recolorization via the sparsity promoting method based on wavelet discretization, and on the bottom-right the result due to the method based on total variation minimization. 

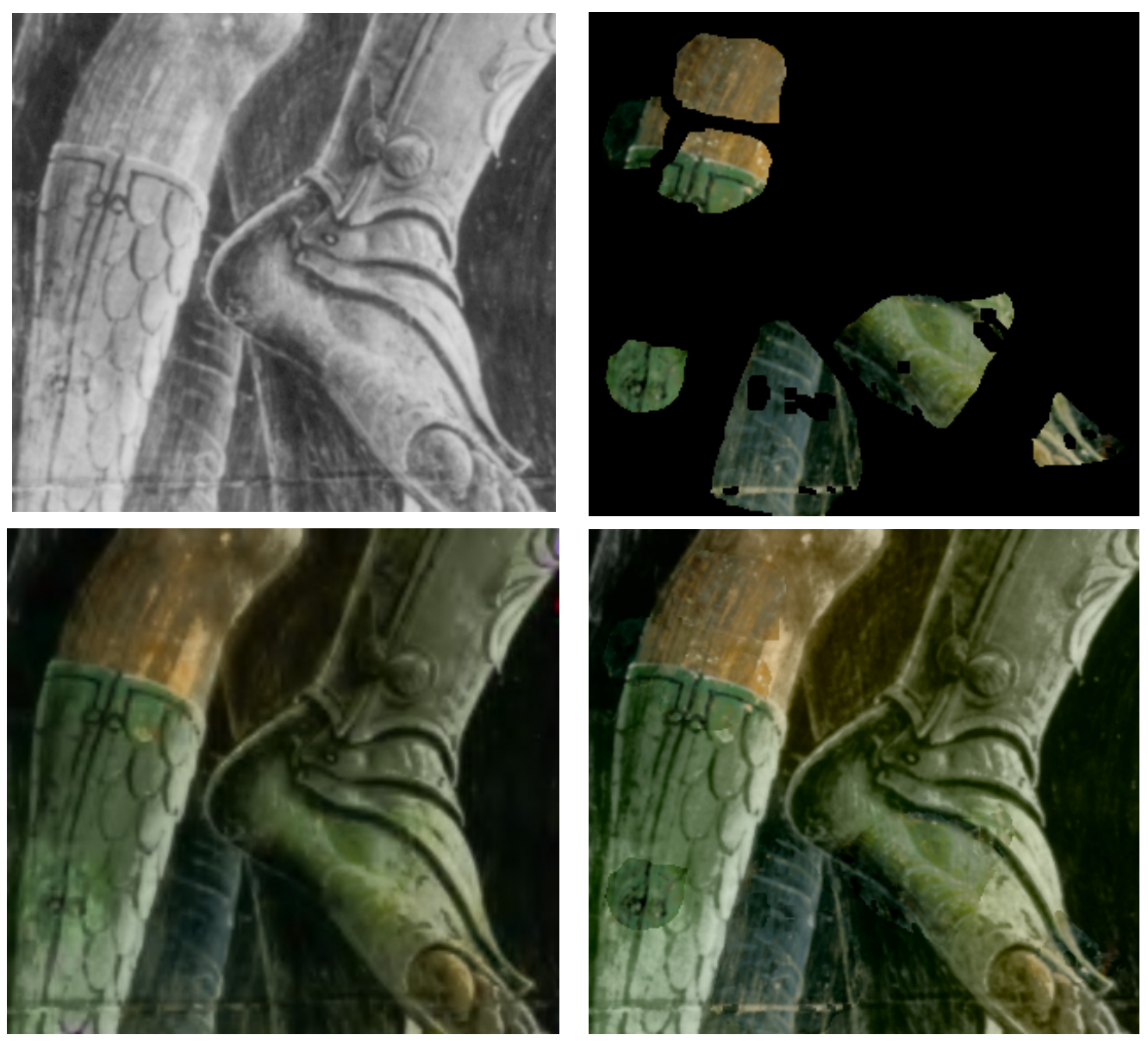

Figure 6: On the top of the figure we show the data, i.e., the gray level and the colored fragments. On the bottom-left we show the result of the recolorization via the sparsity promoting method based on wavelet discretization, and on the bottom-right the result due to the method based on total variation minimization. 
$\mathrm{R}$

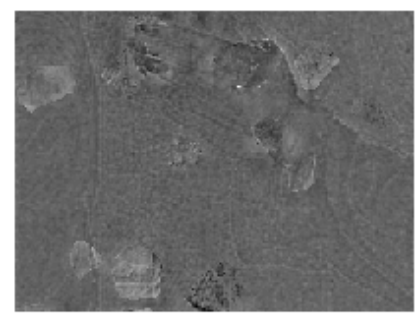

L: light

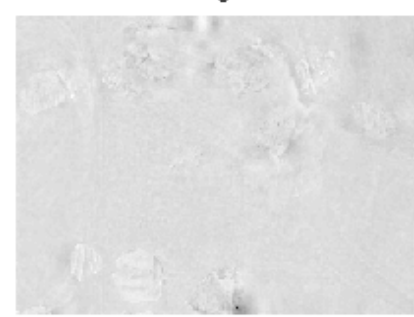

G

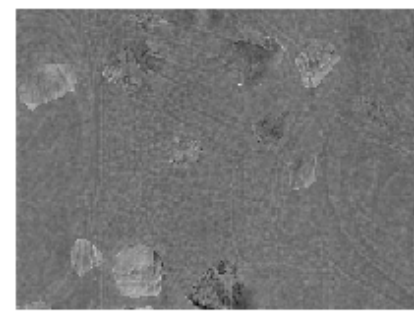

a: green-red

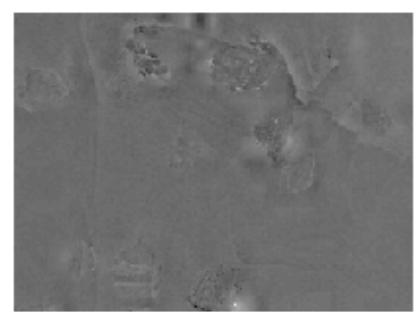

B

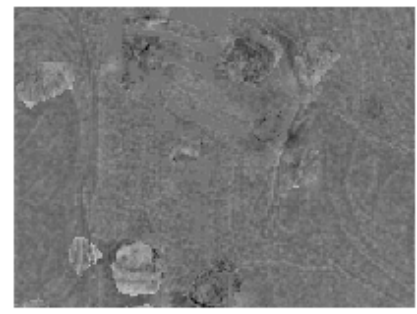

b: yellow-blue

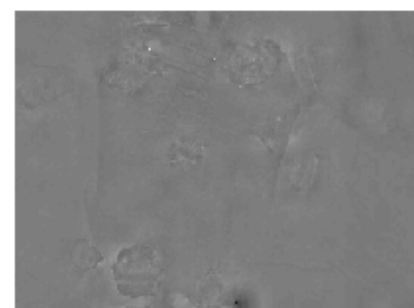

Figure 7: Mutual differences of the first example (see Figure 5) in red, green, and blue channels of the recolorized images by the means two methods (first row) and in the $L, a, b$ channels (second row).

$$
\mathrm{R}^{2}+\mathrm{G}^{2}+\mathrm{B}^{2}
$$

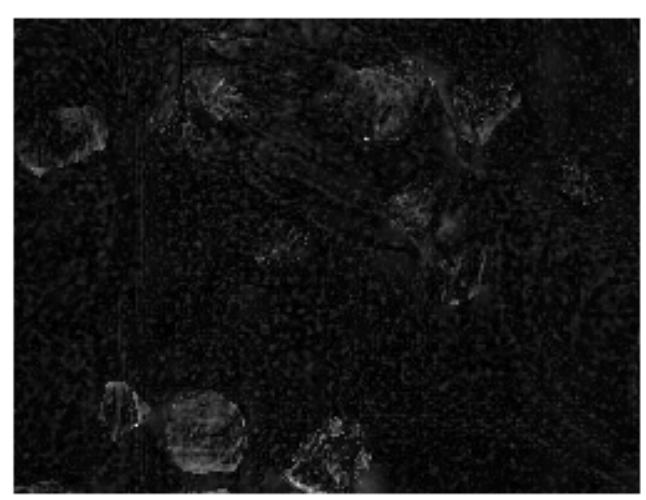

$a^{2}+b^{2}$

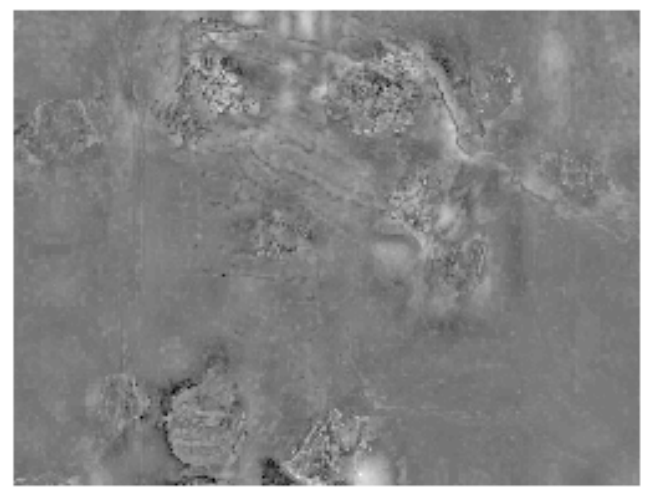

Figure 8: Squared mean of mutual differences of the first example (see Figure 5) in red, green, and blue representation (left) and Squared mean of mutual differences with respect to $a$ and $b$ channels (right). 
$\mathrm{R}$

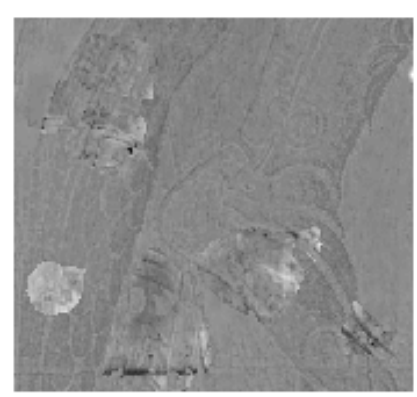

L: light

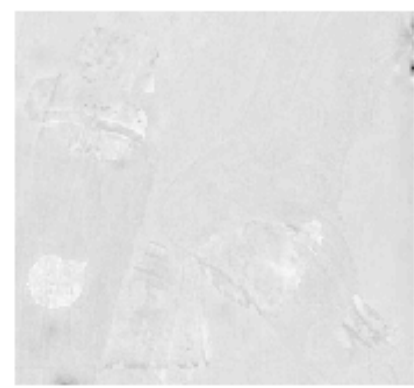

G

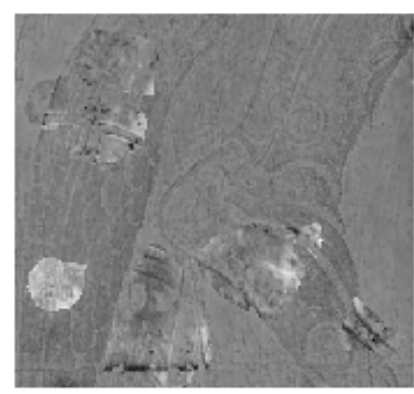

a: green-red

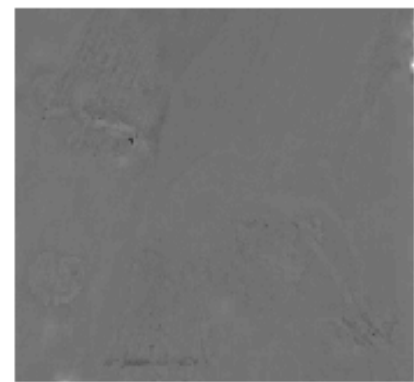

$\mathrm{B}$

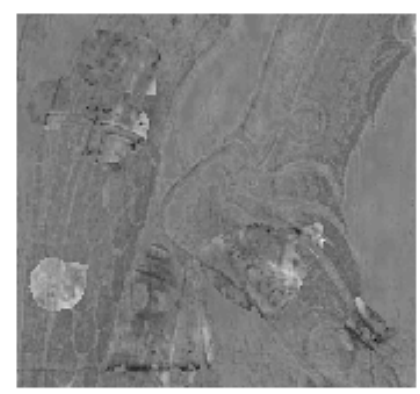

b: yellow-blue

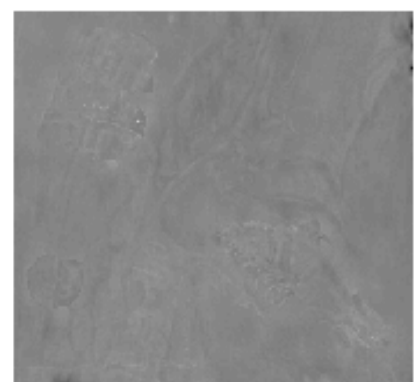

Figure 9: Mutual differences of the first example (see Figure 6) in red, green, and blue channels of the recolorized images by the means two methods (first row) and in the $L, a, b$ channels (second row).

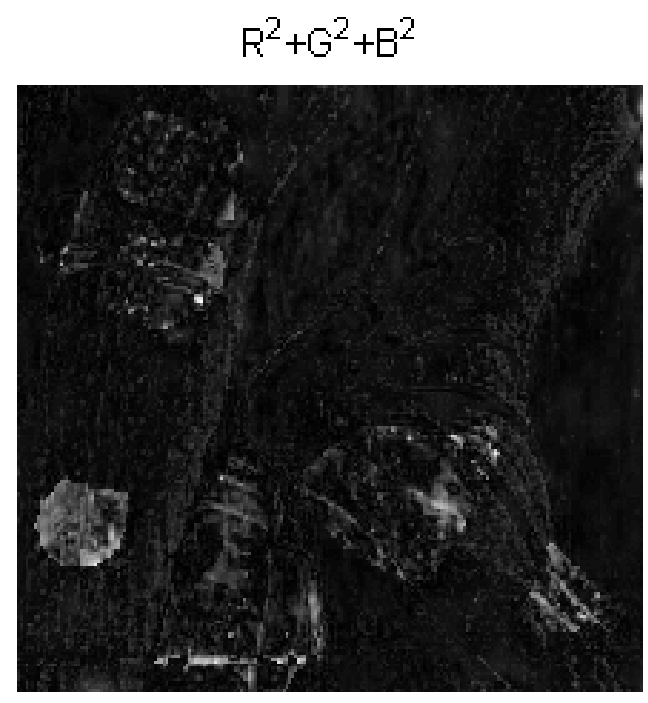

$$
a^{2}+b^{2}
$$

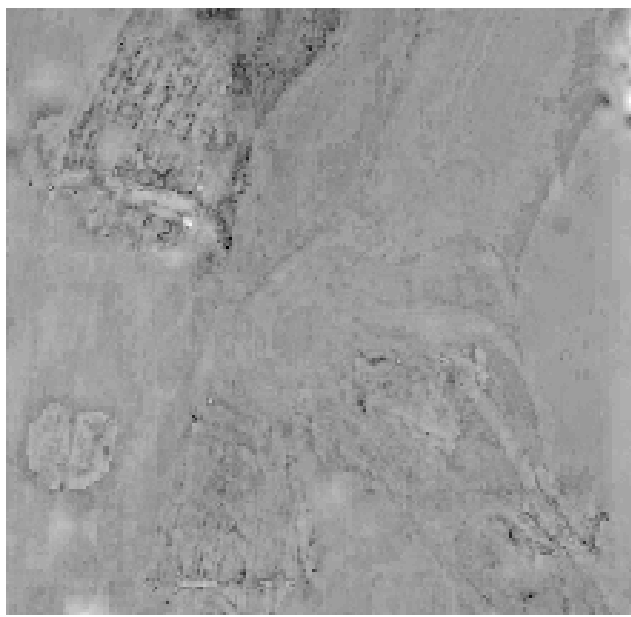

Figure 10: Squared mean of mutual differences of the first example (see Figure 6) in red, green, and blue representation (left) and Squared mean of mutual differences with respect to $a$ and $b$ channels (right). 

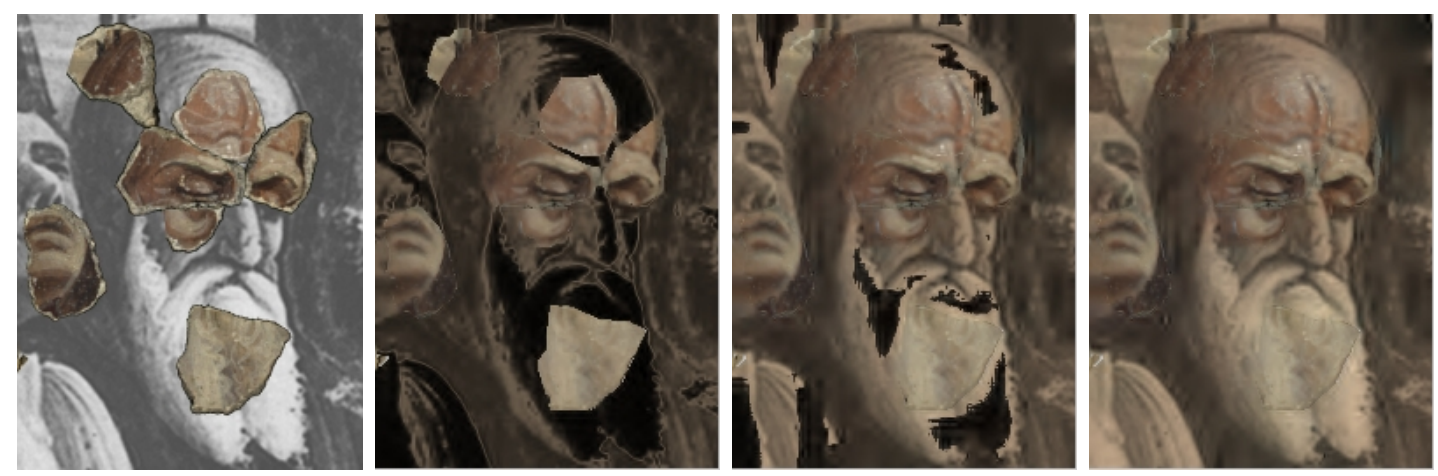

Figure 11: We illustrate a recolorization (three iterates of the WAVELET-JOINT-SPARSE algorithm) of a part of Figure 1. In this case the color-to-gray conversion map cannot be very well estimated due to a limited color palette furnished by the few localized fragments. The result is less satisfactory, but still very important in terms of giving a seeming impression of the original frescoes. 